Ping Liu and Michele A. Basso

J Neurophysiol 100:1098-1112, 2008. First published Jun 25, 2008; doi:10.1152/jn.01043.2007

You might find this additional information useful...

This article cites 87 articles, 39 of which you can access free at:

http://jn.physiology.org/cgi/content/full/100/2/1098\#BIBL

Updated information and services including high-resolution figures, can be found at:

http://jn.physiology.org/cgi/content/full/100/2/1098

Additional material and information about Journal of Neurophysiology can be found at: http://www.the-aps.org/publications/jn

This information is current as of March 16, 2009. 


\title{
Substantia Nigra Stimulation Influences Monkey Superior Colliculus Neuronal Activity Bilaterally
}

\author{
Ping Liu ${ }^{1}$ and Michele A. Basso ${ }^{1,2}$ \\ ${ }^{1}$ Departments of Physiology and ${ }^{2}$ Ophthalmology and Visual Sciences, School of Medicine and Public Health, University \\ of Wisconsin-Madison, Madison, Wisconsin
}

Submitted 20 September 2007; accepted in final form 21 June 2008

Liu P, Basso MA. Substantia nigra stimulation influences monkey superior colliculus neuronal activity bilaterally. J Neurophysiol 100: 1098-1112, 2008. First published June 25, 2008; doi:10.1152/jn.01043.2007. The inhibitory drive arising from the basal ganglia is thought to prevent the occurrence of orienting movements of the eyes, head, and body in monkeys and other mammals. The direct projection from the substantia nigra pars reticulata $(\mathrm{SNr})$ to the superior colliculus $(\mathrm{SC})$ mediates the inhibition. Since the original experiments in the $\mathrm{SNr}$ of monkeys the buildup or prelude neuron has been a focus of SC research. However, whether the $\mathrm{SNr}$ influences buildup neurons in $\mathrm{SC}$ is unknown. Furthermore, a contralateral $\mathrm{SNr}-\mathrm{SC}$ pathway is evident in many species but remains unexplored in the alert monkey. Here we introduced electrical stimulation of one or both $\mathrm{SNr}$ nuclei while recording from SC buildup neurons. Stimulation of the $\mathrm{SNr}$ reduced the discharge rate of SC buildup neurons bilaterally. This result is consistent with activation of an inhibitory drive from SNr to SC. The time course of the influence of ipsilateral $\mathrm{SNr}$ on the activity of most $\mathrm{SC}$ neurons was longer $(\sim 73 \mathrm{~ms})$ than the influence of the contralateral $\mathrm{SNr}(\sim 34 \mathrm{~ms})$. We also found that the variability of saccade onset time and saccade direction was altered with electrical stimulation of the SNr. Taken together our results show that electrical stimulation activates the inhibitory output of the $\mathrm{SNr}$ that in turn, reduces the activity of SC buildup neurons in both hemispheres. However, rather than acting as a gate for saccade initiation, the results suggest that the influence of $\mathrm{SNr}$ inhibition on visually guided saccades is more subtle, shaping the balance of excitation and inhibition across the SC.

\section{N T R O D U C T I O N}

Cortical signals relaying eye movement information to the midbrain traverse at least two pathways. One arises from cerebral cortical neurons and directly targets the superior colliculus (SC) (Fries 1984; Harting et al. 1992; Stanton et al. 1988b). The cortical neurons are excitatory and convey the results of their processing to the SC for the generation of volitional saccades (Segraves and Goldberg 1987; Sommer and Wurtz 2000). A second pathway also arises from neurons of the cerebral cortex and targets the SC through a series of synapses within basal ganglia (BG) nuclei (Hikosaka et al. 1993; Parthasarathy et al. 1992; Selemon and Goldman-Rakic 1985, 1988; Stanton et al. 1988a; Weyand and Gafka 1998). Of the two output nuclei of the BG, oculomotor studies in monkey emphasize the substantia nigra pars reticulata (SNr) (Basso and Liu 2007; Basso and Wurtz 2002; Basso et al. 2005; Bayer et al. 2002; Handel and Glimcher 1999, 2000; Hikosaka and Wurtz 1983a,b,c,d; Sato and Hikosaka 2002) due to its direct projections to the SC (e.g., Jayaraman et al. 1977).

Address for reprint requests and other correspondence: M. A. Basso, Department of Physiology, University of Wisconsin-Madison, Medical School, 1300 University Ave., Room 127 SM1, Madison, WI 53706 (E-mail: michele@physiology.wisc.edu).
Using antidromic stimulation of the $\mathrm{SC}$ and recording of $\mathrm{SNr}$ neurons, a relationship between $\mathrm{SNr}$ neurons that pause transiently and SC neurons that discharge robustly during saccades was established in the monkey (Hikosaka and Wurtz 1983d). These experimental results led to the hypothesis that $\mathrm{SNr}$ inhibits the SC tonically, preventing the occurrence of saccades. The transient pause in $\mathrm{SNr}$ neurons is thought to remove the tonic inhibition from SC neurons, thereby gating the burst of activity within the SC. This series of events results in the initiation of a saccade. Consistent with this, experiments in rodents, cats, and monkeys reveal that disinhibition is a mechanism by which the SNr influences SC (Boussaoud and Joseph 1985; Chevalier and Deniau 1990; Chevalier et al. 1985; Hikosaka and Wurtz 1985a,b; Joseph and Boussaoud 1985).

Since the original experiments in monkeys, a second class of neuron within the monkey SC was characterized more fully (Glimcher and Sparks 1992; Munoz and Wurtz 1995; Sparks 1975). This class of neuron appears distinct from the saccaderelated burst neuron in that it has a low level of tonic discharge well in advance of the burst of action potentials thought to initiate the saccade. Such neurons have been referred to as prelude or buildup neurons (Glimcher and Sparks 1992; Munoz and Wurtz 1995). The low-level, tonic discharge appears while monkeys wait for a cue to make a saccade and is associated with processes such as saccade selection, target selection, attention, movement preparation, and even decision making (Basso and Wurtz 1997, 1998; Glimcher and Sparks 1992; Horwitz and Newsome 1999, 2001; Ignashchenkova et al. 2004; Kim and Basso 2008; Krauzlis and Dill 2002; Kustov and Robinson 1996; McPeek and Keller 2002; Ratcliff et al. 2003). Recent evidence exploring the role of the BG in reward points toward a possible role of the $\mathrm{SNr}$ in modulation of the tonic activity of SC buildup neurons (Hikosaka et al. 2006; Sato and Hikosaka 2002), but whether SNr can influence buildup neurons in monkey SC is unknown. Orthodromic stimulation experiments in the anesthetized cat show that $\mathrm{SNr}$ influences tecto-reticulospinal neurons (Karabelas and Moschovakis 1985), a subclass of which is likely to be the cat homolog of the monkey buildup neuron (Munoz and Wurtz 1995; Rodgers et al. 2006). Recording experiments in SNr, however, show that decreases in tonic activity of $\mathrm{SNr}$ neurons do not exactly mirror the increases in tonic activity of SC neurons. For example, although SC neuronal activity scales approximately linearly with saccade likelihood (Basso and Wurtz 2002; Dorris and Munoz 1998), the tonic activity of SNr

The costs of publication of this article were defrayed in part by the payment of page charges. The article must therefore be hereby marked "advertisement" in accordance with 18 U.S.C. Section 1734 solely to indicate this fact. 
neurons does not (Basso and Wurtz 2002). Therefore the first goal of the present work was to test the hypothesis that $\mathrm{SNr}$ influenced the activity of SC buildup neurons. Our second goal was to determine whether the $\mathrm{SNr}$ influenced buildup neurons in the contralateral SC. Recent experiments in cat, combined with anatomical evidence in rat, cat, and monkey indicate that the SNr targets the contralateral SC (Beckstead 1983; Gerfen et al. 1982; Jiang et al. 2003; Redgrave et al. 1992), but this pathway has not been explored physiologically in the monkey.

Here we used stimulation of the $\mathrm{SNr}$ and recording of SC neurons to test whether alterations of $\mathrm{SNr}$ activity influenced buildup neuronal activity. To drive SC neurons maximally monkeys performed visually guided saccades. Importantly, we used an on-line, spectral filter to remove the stimulus artifact (Gnadt et al. 2003; Paul and Gnadt 2003), so were able to record SC neurons throughout the duration of the electrical stimulus train. When electrical stimulation of the $\mathrm{SNr}$ occurred at the time a cue to make a saccade appeared, SC buildup activity was reduced for the duration of the stimulation. Neurons recorded on the same side as the $\mathrm{SNr}$ stimulation as well as those recorded on the opposite side of the stimulated $\mathrm{SNr}$ showed a reduced rate of discharge during the stimulus train. Based on our results we conclude that the $\mathrm{SNr}$ influences buildup neurons within the monkey SC bilaterally.

\section{METHODS}

\section{General behavioral procedures}

We used a real-time experimental data acquisition and a visual stimulus generation system (Tempo and VideoSync; Reflective Computing) to create the behavioral paradigm and acquire eye position and neuronal data. Trained monkeys sat in a custom-designed primate chair with head fixed during the experimental session (typically 3-5 h). Visual stimuli were rear-projected onto a tangent screen at $51-\mathrm{cm}$ distance using a DLP projector (LP335; Infocus) with a native resolution of $1,024 \times 768$ and operating at $60 \mathrm{~Hz}$. The background luminance was $0.28 \mathrm{~cd} / \mathrm{m}^{2}$. The visual stimulus presentation was controlled by VideoSync software (Reflective Computing) running on a dedicated PC with a $1,024 \times 768$ VGA video controller (Computer Boards). The video PC was a slave to the PC used for experimental control and data acquisition. Two photocells attached to the screen provided accurate measurements of stimulus onset and offset.

Monkeys performed visually guided delayed saccades (Fig. 1, $A$ and $B$ ). Initially a centrally located visual spot appeared and monkeys fixated this spot. After a random time of $500-1,500 \mathrm{~ms}$, a peripheral spot appeared in the visual field. After another delay (800-1,200 ms), the fixation spot disappeared. At this time, monkeys were required to initiate a saccade to the visual spot located in the periphery (Fig. 1, A and $B$ ). When monkeys performed a trial correctly they received a drop of water or fruit juice as reward.

\section{Surgical procedures}

For electrophysiological recording of single neurons and monitoring eye movements, cylinders and eye loops were implanted in four rhesus monkeys (Macaca mulatta) using procedures described previously (Basso and Liu 2007). Anesthesia was induced initially with an intramuscular injection of ketamine $(5.0-15.0 \mathrm{mg} / \mathrm{kg})$. Intramuscular injection of atropine $(0.5 \mathrm{mg} / \mathrm{kg})$ minimized salivation. Monkeys were intubated and maintained at a general anesthetic level with isoflurane. An eye-movement-monitoring device was implanted (Judge et al. 1980). A plastic head holder for restraint and three cylinders (2 SNr and $1 \mathrm{SC}$ ) for subsequent microelectrode recording and stimulation were mounted on top of the exposed skull over craniotomies and
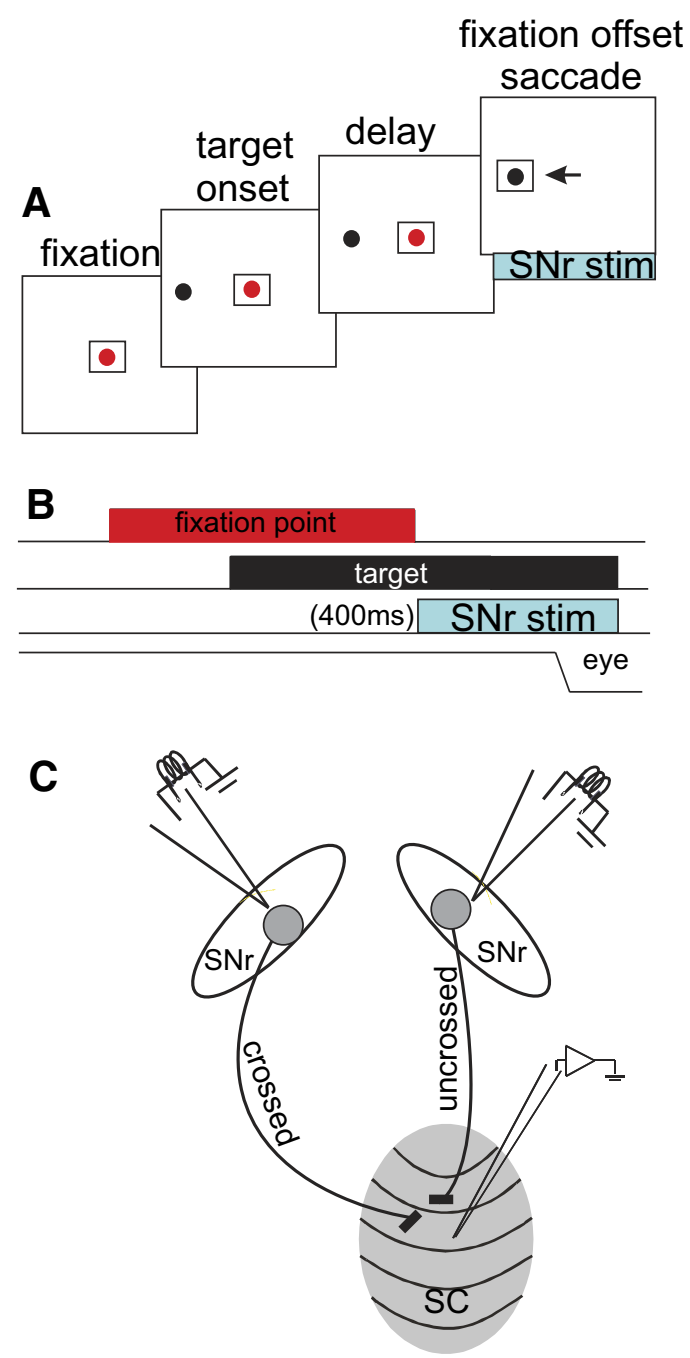

FIG. 1. Behavioral, stimulation, and recording procedures. A: a schematic depiction of the spatial arrangement of the task. Each square indicates the screen at which the monkeys looked. The red spot is the fixation spot and the black spot is the target. The example shows a visually guided saccade made to the left hemifield. The arrow indicates the saccade. The blue rectangle below the labeled "SNr stim" indicates the part of the task in which stimulation of the substantia nigra pars reticulata (SNr) occurred. This occurred coincident with the fixation point offset. $B$ : the temporal arrangement of the visually guided, delayed-saccade task. The $\mathrm{SNr}$ stimulation indicated by the blue rectangle lasted for $400 \mathrm{~ms}$ beginning at the onset of the fixation spot offset. The line labeled "eye" is a schematic of the eye position. $C$ : schematic arrangement of the physiological procedures. See METHODs for details. The ellipses are schematics of left and right $\mathrm{SNr}$ nuclei. The gray oval is a schematic of the right superior colliculus (SC). The $2 \mathrm{SNr}$ nuclei were stimulated (independently) while neurons in the SC were recorded independently; "crossed indicates the $\mathrm{SC}$ on the side contralateral to the stimulation $\mathrm{SNr}$ and "uncrossed" indicates the SC on the side ipsilateral to the stimulation SNr.

secured with titanium screws and dental acrylic. Plastic hardware allowed subsequent magnetic resonance (MR) images to be obtained with minimal artifact. For access to the $\mathrm{SNr}$, the two recording cylinders were targeted toward stereotaxic coordinates A10, L5 and angled mediolaterally about $40-45^{\circ}$, depending on the angle of the $\mathrm{SNr}$ as determined by presurgical MR images. An antibiotic (cefadroxil, $25 \mathrm{mg} / \mathrm{kg}$ ) was given 1 day before and each day for a minimum of 4 days after the operation. For access to both SCs, one cylinder was angled caudorostrally at about $38^{\circ}$ and targeted toward coordinates A0-2, L0. Buprenorphine $(0.01-0.03 \mathrm{mg} / \mathrm{kg}$ ) and flunixin (1-2 mg/kg) were administered $48 \mathrm{~h}$ postsurgically, as needed, to provide analgesia. Monkeys recovered for 1-2 wk 
before experiments commenced. All experimental protocols were approved by the University of Wisconsin-Madison Institutional Animal Care and Use Committee and complied with or exceeded standards set by the Public Health Service policy on the humane care and use of laboratory animals.

\section{Stimulation and recording procedures}

For each experiment, electrodes were aimed at the $\mathrm{SC}$ and $\mathrm{SNr}$ through stainless steel guide tubes held in place by a plastic grid secured to the cylinder (Crist et al. 1988). In a typical experiment, an electrode was advanced each day into the visual-oculomotor region of the SNr (Basso and Wurtz 2002; Handel and Glimcher 1999; Hikosaka and Wurtz 1983d). This region is very small, generally extending $1 \mathrm{~mm} \mathrm{AP} \mathrm{(anterior-posterior} \mathrm{plane)} \mathrm{and} 1 \mathrm{~mm}$ ML (medial-lateral plane). With our cylinders angled at $40^{\circ}$ laterally, we often traverse the entire dorsal-ventral extent of the $\mathrm{SNr}$, which can be as long as 4 $\mathrm{mm}$. Using the grid system for recording (Crist et al. 1988) the visual-oculomotor region is usually found in only one or two grid holes (separated by $1 \mathrm{~mm}$ ML). In our experience, the different $\mathrm{SNr}$ neuronal response profiles are intermingled throughout this region (Basso and Liu 2007; Handel and Glimcher 1999).

Microelectrodes were introduced into each $\mathrm{SNr}$ first. Once an $\mathrm{SNr}$ neuron was isolated, we mapped the response field (RF) by having monkeys make visually guided saccades to six different locations $(0$, 180 , and $45^{\circ}$ up and down, left and right, excluding the directly upward and downward locations). We then introduced a third microelectrode into the SC (uncrossed, Fig. $1 C$ ), isolated a neuron, and assessed its RF by moving a visual spot throughout the visual field, having monkeys make saccades to the same locations and listening for the maximal discharge. These experiments are difficult and RF mapping is time consuming, so we opted to assess SC fields qualitatively because they are easier to determine on-line compared with SNr. Once the center of the SC RF was identified, monkeys performed about 10-12 saccades to that location in the delayed-saccade task to classify the neuron type. With the SC neuron isolated we then introduced electrical stimulation to the $\mathrm{SNr}$ in the same hemifield (uncrossed, Fig. $1 C$ ). Using tungsten microelectrodes (FHC) with impedances $<0.30$ $\mathrm{m} \Omega$, we introduced electrical stimulation of the $\mathrm{SNr}$ at the time the fixation spot was removed. The stimulation train continued for 400 ms. We stimulated regions of the $\mathrm{SNr}$ in which we found clear visual, delay, and/or saccade-related activity. Electrical stimulation parameters were $300 \mathrm{~Hz}$, biphasic pulses each with 150- to 200- $\mu \mathrm{S}$ duration, and a maximum intensity of $60 \mu \mathrm{A}$. We often found that stimulation intensities about $80 \mu \mathrm{A}$ produced shoulder twitches. We interpreted this as current spread into the adjacent internal capsule and therefore did not exceed $60 \mu \mathrm{A}$. In previous experiments we manipulated the frequency of the electrical stimulation among 75, 125, and $300 \mathrm{~Hz}$ and assessed the influence on saccades. Although all frequencies were effective, higher frequencies often produced more reliable results (Basso and Liu 2007). A Grass S88 dual-output square-wave pulse generator provided the input driving two PSIU6s (photoelectric stimulus isolation units). The PSIUs each produced one phase of a biphasic pulse with constant current. For safety, these units are optically isolated (Grass Technologies, AstroMed). The pulses in the stimulation trains were current balanced to minimize tissue damage (Asanuma and Arnold 1975). To ensure accurate current intensities, we measured the current before and after stimulation experiments on an oscilloscope using a 10- $\Omega$ resistor in series with the stimulating electrode.

With the SNr-stimulating electrode in place and an SC neuron isolated, we collected a series of delayed-saccade trials in which stimulation of the $\mathrm{SNr}$ occurred on randomly interleaved trials. Once complete, if the isolation of the SC neuron remained, we returned to the second electrode in the opposite $\mathrm{SNr}$ (crossed, Fig. 1C) and performed the same procedure as described earlier. If the isolation of the SC neuron no longer remained, we moved the electrode to isolate another SC neuron and repeated the procedure described earlier.

To remove the stimulation artifact from the neuronal recording we used the Artifact Zapper (Riverbend Instruments). This hardware performs spectral filtering of the recorded signal, allowing an artifactfree trace of the neuronal waveform (Gnadt et al. 2003; Paul and Gnadt 2003). For each experiment we monitored the unfiltered and the filtered waveforms independently on separate channels of an oscilloscope to ensure successful artifact removal on each trial. Since the device requires an "image" of the stimulus artifact to perform the artifact subtraction, a series of stimulation trials were presented in order for the image acquisition. During this time we were vigilant about not driving the isolated SC neuron, ensuring confidence that SC neuronal waveforms were not included in the subtracted artifact image. Figure 2 shows an example set of spike trains from one
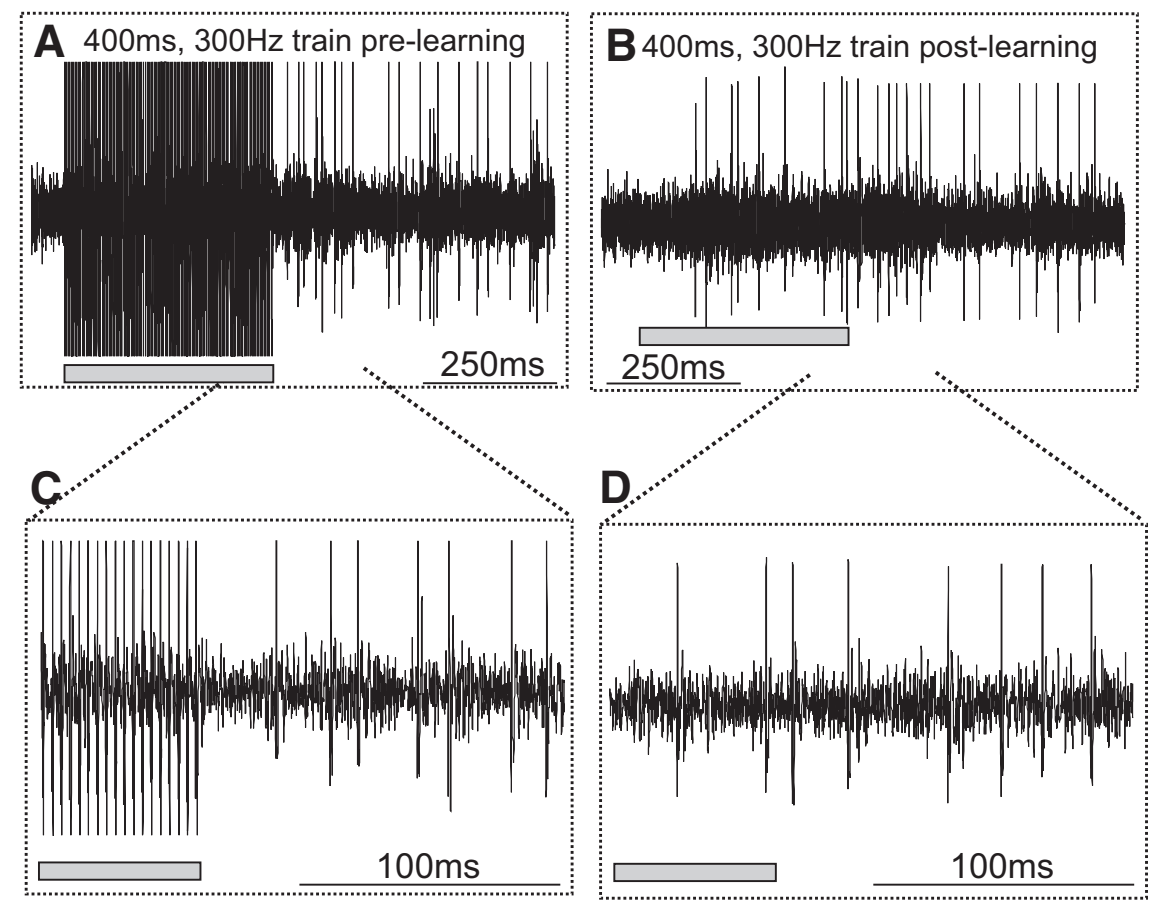

FIG. 2. Examples of spike trains before and after stimulus artifact learning. A: $1,000-\mathrm{ms}$ sweep of the voltage trace (in analog-to-digital $[\mathrm{A} / \mathrm{D}]$ units) plotted against time. A $300-\mathrm{Hz}, 50-\mu \mathrm{A}$ train of pulses recorded as artifact from the electrode in the SC. $B: 1,000-\mathrm{ms}$ sweep of the voltage trace from the same recording day but a different trial after the Artifact Zapper learned the stimulus artifact. $C$ : the same recording as shown in $A$ in expanded view. Only 18 pulses $(60 \mathrm{~ms})$ of the $300-\mathrm{Hz}$, 400-ms stimulus train are seen. $D$ : the same trace as in $B$ expanded in time. The oblique dotted lines indicate the regions of the top traces that were expanded in time. The filled gray rectangles show the regions of the traces where the artifact occurred. There is a small amount of temporal jitter between the onset of the stimulation train shown in $B$ and $C$ compared with the onset shown in $A$ and $D$ because the exact time of the onset of the stimulation varied across trials.

$J$ Neurophysiol • VOL $100 \bullet$ AUGUST $2008 \cdot$ www.jn.org 
recording within the SC with and without stimulation. Figure 2, $A$ and $C$ shows a spike train before the zapper learned the artifact. Figure 2, $B$ and $D$ shows a train after the learning and removal of the artifact.

\section{Data acquisition}

Single neurons were recorded with tungsten microelectrodes (FHC) with impedances between 0.1 and $1.0 \mathrm{M} \Omega$ measured at $1 \mathrm{kHz}$. Action potential waveforms were identified with a window discriminator (Bak Electronics) that returned a TTL pulse for each waveform meeting voltage and time criteria. The waveform discriminator (Bak Electronics) was placed in series with the Artifact Zapper (Riverbend Instruments) and waveform discrimination occurred after removal of the stimulus artifact. In some albeit rare cases, we could isolate single waveforms independent of the stimulus artifact removal system, providing a second way to ensure that the stimulation artifact did not contaminate the spike train data (Anderson et al. 2003). The TTL pulses were sent to a digital counter (PC-TIO-10; National Instruments) and were stored with a 1-ms resolution. Once an $\mathrm{SNr}$ neuron was isolated and characterized in the delayed-saccade task, using the same electrode, electrical stimulation commenced. By this time, the electrodes had impedances between 100 and $300 \mathrm{k} \Omega$. For eye movement recording, we used the magnetic induction technique (Fuchs and Robinson 1966) (Riverbend Instruments). Voltage signals proportional to horizontal and vertical components of eye position were filtered (eight-pole Bessel; $-3 \mathrm{~dB}, 180 \mathrm{~Hz}$ ), digitized at 16-bit resolution, and sampled at $1 \mathrm{kHz}$ (CIO-DAS1602/16; Measurement Computing). The analog eye position data were saved for off-line analysis using an interactive computer program designed to display and measure eye position and calculate eye velocity. We used an automated procedure to define saccadic eye movements by applying velocity and acceleration criteria of $25 \%$ and $8,000 \% \mathrm{~s}^{2}$, respectively. The result of the algorithm was confirmed or corrected if necessary on a trial-by-trial basis by the experimenter.

\section{Neuronal classification}

Previous work in the cat suggests that there is a topography of $\mathrm{SNr}$ response types within the $\mathrm{SNr}$ projecting to SC. To see whether we could find a similar topography in our experiments, we kept a careful record of the $\mathrm{SNr}$ response types recorded at each stimulation site. $\mathrm{SNr}$ neurons were classified as visual, saccade, or visual-delaysaccade as done previously (Basso and Liu 2007). For classification, we measured $200 \mathrm{~ms}$ of baseline discharge rate while monkeys fixated and before a visual stimulus appeared. We then measured the first 200 ms of neuronal discharge after the onset of the visual stimulus. The delay interval was defined as the discharge rate occurring 600-800 $\mathrm{ms}$ after the target spot appeared. The saccade interval was defined as the discharge rate occurring $50 \mathrm{~ms}$ before to $50 \mathrm{~ms}$ after the onset of the saccade. $\mathrm{SNr}$ neurons were classified as visual if they contained statistically significant differences $(t$-test, $P<0.05)$ in discharge rate during the visual interval compared with baseline. Saccade neurons were defined as those showing a significant difference in discharge rate during the saccade interval compared with baseline ( $t$-test, $P<$ 0.05). Visual-delay-saccade neurons were classified as those with statistically significant discharge rates during all three intervals compared with baseline ( $t$-test, $P<0.05)$. Some visual and saccade neurons also had significant modulation during at least one other interval. These were classified as either visual-delay neurons if the delay period modulation was also significant or visual saccade if the saccade period was also significant. Of our sample, five neurons had increases in activity and appeared like those described as pause-burst neurons (Handel and Glimcher 1999). In our classification scheme, two of five were visual-delay-saccade neurons, two of five were visual neurons, and one of five was a visual-delay neuron. Consistent with a lack of topography, the effects of stimulation at sites where these neurons were recorded were not obviously different from the effects observed at locations where other neuronal response profiles were found.

SC neurons were classified as burst (visual motor) or buildup. Using data from correct trials of the visually guided delayed-saccade task we computed a baseline interval (average discharge rate -200 to $0 \mathrm{~ms}$ before the onset of the stimuli), a visual interval $(0-200 \mathrm{~ms}$ beginning at target onset), a delay interval (300-800 ms after the target onset), and a saccade interval ( -50 to $0 \mathrm{~ms}$ before the saccade onset). Buildup neurons had significantly greater activity in the delay interval compared with the baseline ( $t$-test, $P<0.05)$ and significantly greater activity in the saccade interval compared with the delay interval ( $t$-test, $P<0.05$ ). Burst or visual motor neurons had no statistically significant delay activity compared with baseline but had significant increases in discharge during the saccade interval compared with baseline.

\section{Data analysis}

All statistical analyses were performed using Matlab (MathWorks). We made statistical comparisons using parametric ANOVA or $t$-test (modified-Bonferroni methods). If the data failed to pass normality tests, nonparametric ANOVA (Kruskal-Wallis) or Wilcoxon ranksum tests were used (Keppel 1991).

To compare the time course of SC neuronal activity changes with $\mathrm{SNr}$ stimulation, we computed receiver operating characteristic (ROC) curves based on signal detection theory (Green and Swets 1966), similar to that performed by others (Bradley et al. 1987; Britten et al. 1992; Cohn et al. 1975; Thompson et al. 1996). Each spike train for each trial was convolved with a Gaussian having a $\sigma=3 \mathrm{~ms}$. We computed the probability that the discharge rate exceeded a criterion for each millisecond of discharge beginning at $100 \mathrm{~ms}$ before the fixation spot removal (stimulation onset) and continuing for $500 \mathrm{~ms}$. This was performed for each neuron in the two sets of trials, stimulation and no stimulation. The criterion was incremented from the minimum to the maximum discharge rate in the epoch in step sizes of (maximum - minimum discharge rate)/100. A probability value was computed for each criterion. A single point on the ROC curve was produced for each increment in the criterion and the entire ROC curve was generated from all the criteria. Rather than relying on an arbitrary ROC area criterion to determine statistical significance, we performed a permutation test (Efron and Tibshirani 1998). For each neuron, we randomly sampled the discharge rate across the epoch length 1,000 times and generated an ROC curve for each permutation. This resulted in a distribution of ROC areas. The original ROC area for individual neurons was compared with this distribution of areas to determine whether it fell within or outside of the 95th percentile. If the original value fell outside of the 95th percentile for $\geq 5 \mathrm{~ms}$ we determined that the difference between the two curves at that time point was statistically reliable. We then plotted the ROC area obtained for each millisecond as a function of time (Fig. 7).

\section{RES U L T S}

While monkeys performed a visually guided delayed-saccade task (Fig. 1, $A$ and $B$ ), we recorded from SC neurons during stimulation of either the contralateral or the ipsilateral $\mathrm{SNr}$ (Fig. 1C). We recorded from eight SCs and stimulated in eight SNrs of four monkeys. Thirty SC neurons were recorded during stimulation of the $\mathrm{SNr}$ in the same hemisphere as the recorded SC neuron (referred to as uncrossed). Twenty SC neurons were recorded during stimulation of the $\mathrm{SNr}$ in the opposite hemisphere as the recorded SC neuron (referred to as crossed). For eight of the uncrossed SC neurons, short, fixedlatency responses appeared with $\mathrm{SNr}$ stimulation. Although we did not perform the collision test (Bishop et al. 1962; Fuller and Schlag 1976), we interpreted this observation as evidence of 
antidromic activation of the tectonigral pathway (Comoli et al. 2003; Karabelas and Moschovakis 1985; York and Faber 1977). Because our interest here concerned orthodromic responses, we excluded these eight neurons from further analysis. The results reported here are from $22 \mathrm{SC}$ neurons recorded with stimulation of the uncrossed SNr-SC pathway and $20 \mathrm{SC}$ neurons recorded with stimulation from the crossed $\mathrm{SNr}-\mathrm{SC}$ pathway. Thirteen of these were the same SC neurons recorded with stimulation of both $\mathrm{SNr}$ nuclei (Fig. 1C). Therefore the data set consists of $29 \mathrm{SC}$ neurons. By recording SC neurons and having monkeys make saccades of the preferred vector for the recorded SC neurons, stimulating the ipsilateral $\mathrm{SNr}$ assessed the influence of the uncrossed pathway. Stimulation of the contralateral $\mathrm{SNr}$ (with respect to the SC) assessed the influence of the crossed pathway.

In what follows, we first describe the overall result of electrical stimulation in $\mathrm{SNr}$ on $\mathrm{SC}$ neurons by illustrating two examples: one uncrossed and one crossed. We then present the results from the sample of recorded neurons and then we present the results showing the influence of $\mathrm{SNr}$ stimulation on the corresponding saccade behavior.

\section{SNr stimulation and SC neuronal activity}

UNCROSSED INFLUENCE. The results from one $\mathrm{SNr}$ stimulation and $\mathrm{SC}$ recording experiment are shown in Fig. 3. As is typical for SC neurons with delay activity (prelude or buildup neurons), this example neuron had a low level of tonic discharge leading up to the time of the cue to make a saccade (Fig. 3A, dashed vertical line). This SC neuron preferred saccades directed to the right hemifield. When aligned on the saccade onset (Fig. $3 B$ ) the robust discharge of action potentials associated with saccade onset was evident. By introducing stimulation of the $\mathrm{SNr}$ at the time of the cue to make a saccade and extending it for $400 \mathrm{~ms}$, we ensured that there was adequate activity of the SC neurons to be influenced by the $\mathrm{SNr}$ stimulation. We took this approach under the premise that with extracellular recordings, the detection of inhibition in neurons with little discharge would be difficult.

Applying electrical stimulation to the $\mathrm{SNr}$ in the same hemisphere as that of the recorded SC neuron resulted in a transient cessation of activity in the SC neuron (Fig. 3C). Within about 100 $\mathrm{ms}$, the SC neuron resumed a high rate of discharge, although this resumed discharge did not reach the same high rate as that seen in the trials without stimulation (Fig. 3, $A$ and $C$ ). This can be seen most clearly in the saccade-aligned traces in which the peak and the duration of the saccade-related burst appeared smaller with $\mathrm{SNr}$ stimulation than without $\mathrm{SNr}$ stimulation (cf., Fig. 3, B and $D$ ). To quantify the change in neuronal activity we measured the mean discharge rate of the SC neuron during a 300-ms window beginning at the time of the disappearance of the fixation spot in the no-stimulation trials (Fig. 3A, gray shaded region). The discharge rate was 72.91 spikes/s in this example. The mean discharge rate of the SC neuron during the same epoch on trials with $\mathrm{SNr}$ stimulation (the stimulation onset and the fixation spot offset occurred simultaneously; Fig. $3 C$, gray shaded region) was 50.22 spikes/s. The difference in discharge rate across trials for this example SC neuron with and without $\mathrm{SNr}$ stimulation was statistically significant $(t$-test, $P<0.01)$. Despite the change in discharge around the time of the saccade, the amplitude of the saccade changed little with electrical stimulation of the $\mathrm{SNr}$ (mean amplitude without stimulation $=10.45^{\circ}$; mean amplitude with

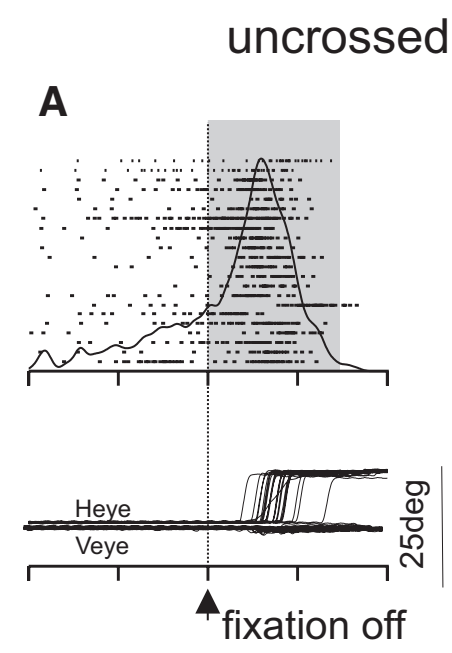

SNr-SC
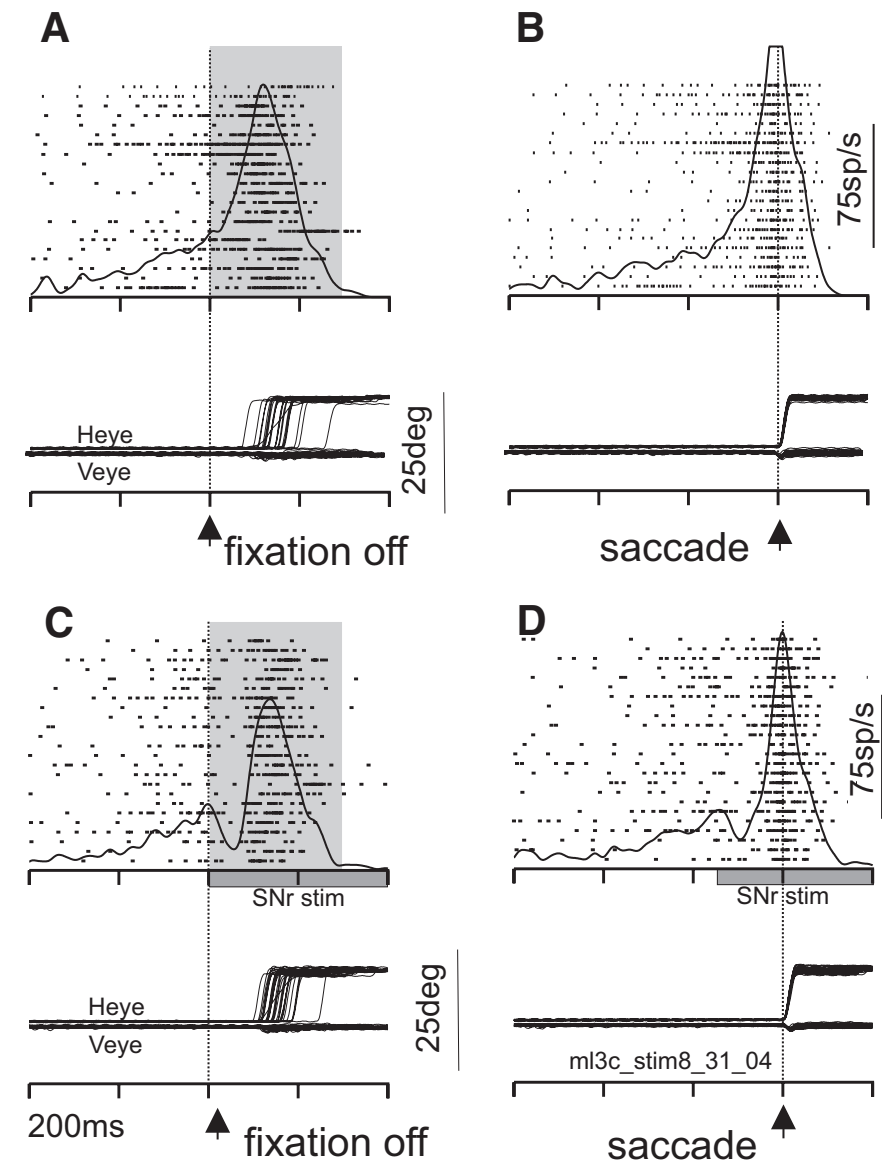

FIG. 3. SNr stimulation suppresses ipsilateral SC neuronal activity. In each panel, each tick indicates the time of occurrence of an action potential and each row indicates a trial in which the monkey made a rightward saccade (raster). The spike density functions ( $\sigma=12 \mathrm{~ms}$ used for display) are superimposed on the rasters. $A$ : example $\mathrm{SC}$ neuron without $\mathrm{SNr}$ stimulation aligned on the offset of the fixation point. The alignment is indicated by the dashed vertical line and the arrowhead at the bottom of the panel. The shading indicates the interval over which discharge rate was measured. $B$ : same neuron as shown in $A$, but aligned on the onset of the saccade. The alignment is indicated by the dashed vertical line and the arrowhead. $C$ : the same neuron as shown in $A$ and $B$, but now for trials in which $\mathrm{SNr}$ stimulation was applied. The traces are aligned on fixation spot offset. The shading indicates the interval over which discharge rate was measured. The stimulation train is indicated by the gray rectangle on the abscissa. $D$ : the same neuron as in $A$ and $B$ now aligned to the onset of the saccade and with $\mathrm{SNr}$ stimulation. The stimulation train is indicated by the gray rectangle on the abscissa. Heye, horizontal eye position; Veye, vertical eye position; fpoff, fixation point offset. Each tick on the time axis is separated by 200 ms (ml3c_stim8_31_04 is the filename used for reference).

stimulation $=10.55^{\circ}$; Wilcoxon, $P=0.77$ ). For this example, $\mathrm{SNr}$ stimulation reduced the average saccade velocity across the trials (mean without stimulation $=581.30^{\circ} / \mathrm{s}$; mean with stimulation $=555.25^{\circ} / \mathrm{s}$; Wilcoxon rank-sum test, $P<0.01$ ). This example provides direct evidence that the $\mathrm{SNr}$ inhibits presaccade and saccade-related discharge of SC buildup neurons and is associated with a reduced velocity of saccades.

CROSSED INFLUENCE. Stimulation of the $\mathrm{SNr}$ in the hemisphere opposite to the recorded SC neuron produced similar results as seen for stimulation within the same hemisphere (Fig. 4). Stimulation of the $\mathrm{SNr}$ opposite the recorded SC produced a transient reduction in the discharge of the SC neuron (Fig. 4, A 


\section{crossed SNr-SC}
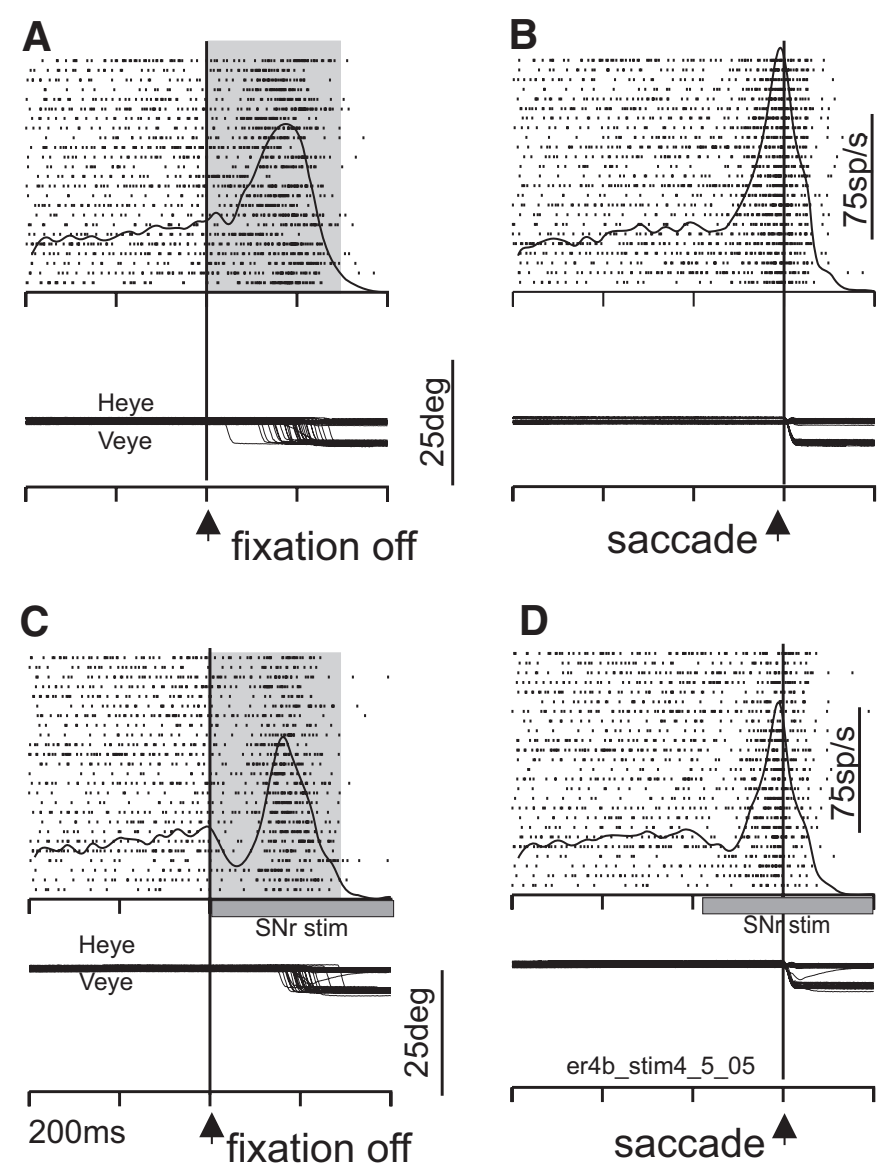

FIG. 4. SNr stimulation suppresses contralateral SC neuronal activity. The arrangement of this figure is the same as Fig. 3. $A$ and $C$ are aligned on fixation point onset. $B$ and $D$ are aligned on saccade onset. $A$ and $B$ are without $\mathrm{SNr}$ stimulation. $C$ and $D$ are with $\mathrm{SNr}$ stimulation. Heye, horizontal eye position; Veye, vertical eye position; fpoff, fixation point offset. Each tick on the time axis is separated by $200 \mathrm{~ms}$ (er4b_stim4_5_05 is the filename used for reference).

and $C$ ). As observed for the uncrossed stimulation, the suppression of neuronal discharge was transient, lasting for about $100 \mathrm{~ms}$. The SC neuron then resumed its high rate of discharge, although not as high as that seen in trials without stimulation. Again this latter aspect of the result is seen more clearly in the saccade-aligned traces (Fig. 4, $B$ and $D$ ). During the 300-ms epoch beginning with the removal of the fixation spot, the mean neuronal discharge rate for this neuron was 106.92 spikes/s without stimulation; during the same epoch the mean discharge rate was 82.65 spikes/s with $\mathrm{SNr}$ stimulation. Across trials these differences in the SC discharge rate were statistically significant $(t$-test, $P<0.01)$. As observed for the uncrossed stimulation example, the amplitude of the visually guided saccades did not differ between trials with or without $\mathrm{SNr}$ stimulation. For the example shown in Fig. 4, the mean of the saccade amplitudes was $-4.79^{\circ}$ in the no-stimulation condition, whereas the mean of the saccade amplitudes in the stimulation condition was $-4.82^{\circ}$ (Wilcoxon, $P=0.59$ ).

Slight differences in the velocities of the saccades in the stimulation and no-stimulation trials occurred in this example. In trials without $\mathrm{SNr}$ stimulation the mean of the radial saccade velocities was $292.76^{\circ}$ s, whereas in trials with $\mathrm{SNr}$ stimulation, the mean of the radial saccade velocities increased slightly to $297.44 \%$ s. These differences failed to reach statistical significance (Wilcoxon, $P=0.71$ ). Taken together, these two examples show that $\mathrm{SNr}$ stimulation inhibits the activity of SC buildup neurons whether stimulation occurs in the same hemisphere as the recorded SC neuron or in the opposite hemisphere. Furthermore, in spite of the significant changes in the discharge rate of the SC neurons, there was little evidence of change in the characteristics of saccades. The latter result is consistent with our previous observations that electrical stimulation of the $\mathrm{SNr}$ had little effect on visually guided saccades (Basso and Liu 2007).

\section{SNr stimulation suppresses SC neuronal activity: group results}

To determine whether the change in discharge rate of SC neurons associated with electrical stimulation of the $\mathrm{SNr}$ occurred consistently across our sample of SC neurons, we measured the discharge rate during a 300-ms interval beginning at the time of the fixation spot offset. This time corresponded to the onset of the electrical stimulation of the SNr. Figure 5 shows the mean of the discharge rates measured across trials for each $\mathrm{SC}$ neuron with $\mathrm{SNr}$ stimulation against the mean of the discharge rates of the $\mathrm{SC}$ neurons without $\mathrm{SNr}$ stimulation. For uncrossed neurons (Fig. 5A), the mean discharge rate without stimulation was 73.80 spikes/s. The mean discharge rate dropped to 64.43 spikes/s with stimulation. The decreases in discharge rate for uncrossed SC neurons between stimulation and no-stimulation trials were individually significant in 9 of $22(41 \%)$ of the neurons (Fig. 5A, filled circles below unity line; $t$-test, $P<0.05$ ). Thus stimulation of the $\mathrm{SNr}$ on the same side as the recorded SC neuron resulted in an overall reduction in $\mathrm{SC}$ neuronal activity.

For crossed neurons (Fig. 5B), the mean discharge rate without stimulation was 73.78 spikes/s. With stimulation of the $\mathrm{SNr}$, the discharge rate dropped to 62.68 spikes/s. The decreases in discharge rate for crossed SC neurons between stimulation and no-stimulation trials were individually significant in 13 of $20(65 \%)$ of the neurons (Fig. 5B, filled circles below unity line; $t$-test $P<0.05$ ). Across the sample of SC neurons regardless of the side of $\mathrm{SNr}$ stimulation, three neurons had slight but statistically significant increases in neuronal activity with $\mathrm{SNr}$ stimulation (Fig. 5, $A$ and $B$, filled circles above the line of unity; $t$-test $P<0.05$ ). Taken together we conclude that $\mathrm{SNr}$ stimulation primarily suppresses SC buildup neuronal activity bilaterally.

Figure 6 shows the average response profiles of uncrossed and crossed SC neurons when aligned on the disappearance of the fixation spot (Fig. 6, $A$ and $C$ ) and when aligned to the onset of the saccade (Fig. 6, $B$ and $D$ ). In the uncrossed neurons as a group, there was a slight reduction in activity followed by an apparent, accelerated rise in the saccade-related burst. The saccade-related burst overall was reduced slightly in the stimulated trials compared with the unstimulated trials (Fig. 6A). When the same data were aligned on the saccade, there was a slight reduction in discharge rate for most of the duration of the stimulus train (Fig. 6B). Even the transient visual activation appearing at the end of the saccade was reduced in the stimulated trials (Fig. 6, $A$ and $B$, arrowheads). The difference 


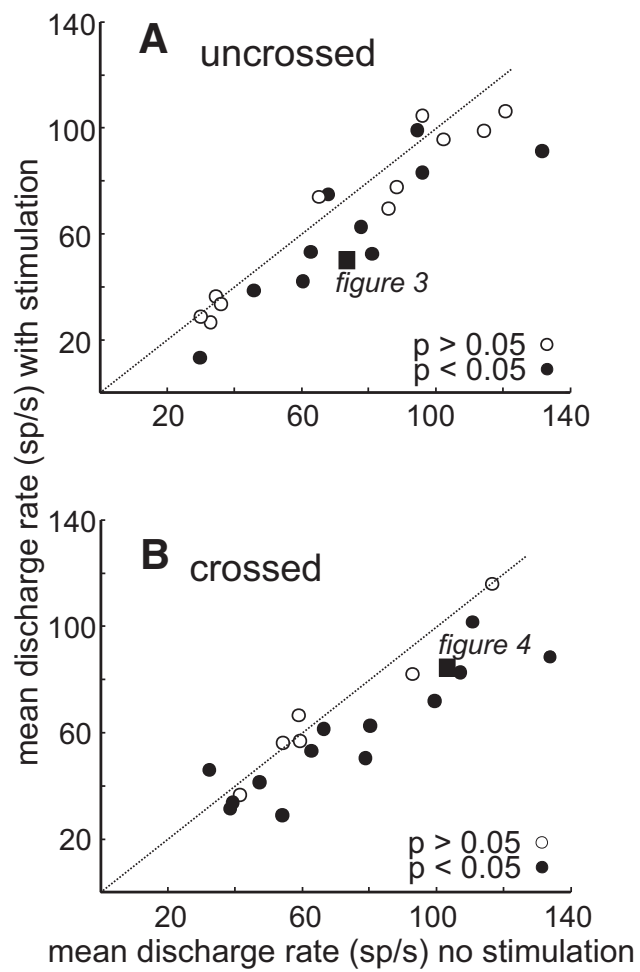

FIG. 5. SNr stimulation primarily suppresses $\mathrm{SC}$ neuronal activity. A: mean discharge rate (spikes/s) with $\mathrm{SNr}$ stimulation plotted against mean discharge rate (spikes/s) without $\mathrm{SNr}$ stimulation for all $\mathrm{SC}$ neurons recorded in the same hemisphere as the $\mathrm{SNr}$ stimulated (uncrossed). The dotted oblique line is the line of unity. Points falling below the line indicate decreased discharge rate (during the 300-ms interval after fixation point offset/stimulation onset) with stimulation. Points above the line indicate increased discharge rate with $\mathrm{SNr}$ stimulation. Filled black circles indicate neurons with statistically significant differences between the stimulation and no-stimulation condition (Wilcoxon, $P<0.05$ ). Unfilled circles indicate no significant difference between discharge rates of $\mathrm{SC}$ neurons with $\mathrm{SNr}$ stimulation (Wilcoxon, $P>0.05$ ). The filled square is from the example neuron shown in Fig. 3. B: same as in $A$ for the SC neurons recorded in the hemisphere opposite the side of $\mathrm{SNr}$ stimulation (crossed). The filled square shows the result for the example neuron shown in Fig. 4.

in neuronal activity measured with and without stimulation of the $\mathrm{SNr}$ across the sample of uncrossed SC neurons was statistically significant (Kruskal-Wallis, $P<0.001$ ).

A similar finding was obtained for $\mathrm{SC}$ neurons recorded in the hemisphere opposite the stimulated $\mathrm{SNr}$ (Fig. 6, $C$ and $D$ ). In this case, however, the reduction in SC activity was more robust (Fig. 6, $A$ and $C$ ). When the same data were aligned on the onset of the saccade, a similar pattern appeared. SC neuronal activity was reduced for most of the duration of the stimulus train and even the postsaccadic visual transient was reduced with $\mathrm{SNr}$ stimulation (Fig. 6, $C$ and $D$, arrowheads). Across the sample of crossed SC neurons, statistically significant suppression of neuronal activity occurred (Kruskal-Wallis, $P<0.001$ ).

Table 1 shows the breakdown of the different SC neuron classes, the different $\mathrm{SNr}$ neuron classes recorded from the sites of stimulation, and the correspondence of the preferred hemifield for both $\mathrm{SNr}$ and SC neurons. The observations can be summarized as follows. First, by design, we recorded mostly from buildup or prelude neurons. When SC recording and $\mathrm{SNr}$ stimulation were in opposite hemispheres, we recorded from two burst neurons. When the $\mathrm{SNr}$ stimulation and the SC recording were in the same hemisphere, we recorded from two burst neurons. In each case, one burst neuron showed reduced neuronal activity with $\mathrm{SNr}$ stimulation and one showed no change. All the rest of the neurons recorded from SC were buildup (prelude) neurons. Second, although most neuron types commonly found in the $\mathrm{SNr}$ appeared in our sample, the majority was the visual-delay-saccade neuron. These neurons most often show decreases in discharge rate for all three epochs of a delayed-saccade task (see METHODS). We found no particular trend between the type of $\mathrm{SNr}$ neuron recorded at the site and the influence of electrical stimulation on the SC neuron. No matter what the response profile of the $\mathrm{SNr}$ neuron at the stimulation site, the influence of stimulation on SC neuronal activity was most likely to be suppression.

Previous studies in cat revealed a relationship between the preferred RF location of $\mathrm{SNr}$ neurons and whether the neuron was part of the crossed or uncrossed pathway (Jiang et al. 2003). Therefore we assessed the RFs of our sample of SNr and SC neurons. We defined the preferred hemifield for SC neurons qualitatively by listening to the discharge while the monkeys made saccades to different target locations. For the $\mathrm{SNr}$, we determined the preferred hemifield statistically by having the monkeys make saccades to six different target positions (see METHODS). The hemifield associated with the largest, statistically significant change in activity was defined as the preferred hemifield. For SC neurons recorded on the same side as the stimulated $\mathrm{SNr}, 18$ of $22(82 \%)$ of the $\mathrm{SNr}-\mathrm{SC}$ pairs had the same preferred hemifield. For the SC neurons recorded from the opposite side of $\mathrm{SNr}$ stimulation, 0 of 22 $(0 \%)$ had the same preferred hemifield; 20 of $20(100 \%)$ of the $\mathrm{SNr}-\mathrm{SC}$ stimulation-recording pairs from opposite sides had opposite preferring hemifields. Only 4 of $22(18 \%)$ of the pairs from the same side had opposite preferring hemifields.

\section{Time course of SNr influence on SC neuronal activity}

We next explored the timing of the influence of $\mathrm{SNr}$ stimulation on SC neuronal activity. Often visual inspection is adequate to determine the time of an increase in neuronal discharge with extracellular recording. Determining the time of decreases in extracellular neuronal discharge however, is less straightforward. We opted to use the statistically rigorous and sensitive method based on signal detection theory (Green and Swets 1966): receiver operating characteristic (ROC) analysis. This method, combined with the fact that we are measuring extracellular action potentials, likely overestimates the actual time the SNr is able to influence SC (Karabelas and Moschovakis 1985). By computing ROC curves combined with a bootstrapping procedure (see METHODS) we determined the time point at which the spike density functions from stimulated trials and nonstimulated trials became significantly different from one another. For this analysis, ROC areas of 0.50 indicate no difference between the stimulated and nonstimulated spike trains; values $>0.50$ indicate increases in activity, whereas values $<0.50$ indicate decreases in activity.

Figure $7 \mathrm{~A}$ shows the results of the ROC analysis performed for all the neurons in our sample. ROC area is plotted against time in milliseconds, beginning $100 \mathrm{~ms}$ before the onset of the $\mathrm{SNr}$ stimulation. The result for the 20 crossed neurons is shown in black and the result for the 22 uncrossed neurons is shown in red. Shortly after the stimulation train began there was a 

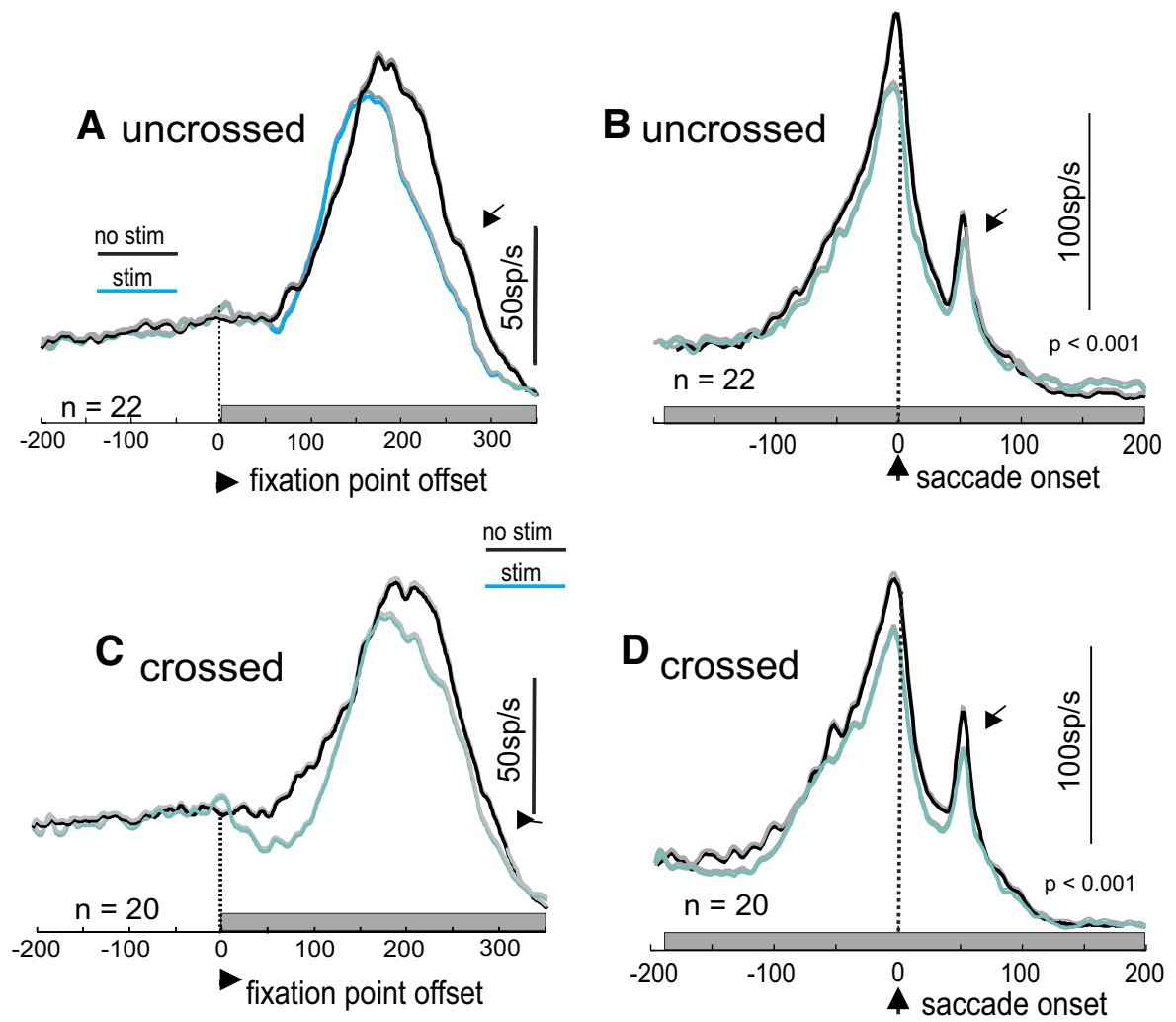

FIG. 6. SNr stimulation suppresses saccade-related discharge of SC neurons. $A$ : the average spike density function ( $\sigma=12 \mathrm{~ms}$ for display) from $22 \mathrm{SC}$ neurons recorded on the same side as $\mathrm{SNr}$ stimulation (uncrossed). Black lines: without stimulation of the SNr; blue lines: with stimulation of the SNr. The thin gray lines (barely visible) are $1 \mathrm{SE}$. The traces are aligned on the fixation point offset indicated by the dashed vertical line and arrowhead at time $0 \mathrm{~ms}$. The gray rectangle indicates the onset and duration of the $\mathrm{SNr}$ stimulation train. $B$ : same neuron as shown in $A$ but now the traces are aligned on the onset of the saccade indicated by the vertical dashed line and the arrowhead at time $0 \mathrm{~ms}$. $C$ : the average spike density function from $20 \mathrm{SC}$ neurons recorded on the opposite side relative to the $\mathrm{SNr}$ stimulation (crossed). Traces are aligned on the fixation point offset (stimulation onset) indicated by the dashed vertical line, arrowhead at time $0 \mathrm{~ms}$. The gray rectangle shows the stimulation train. $D$ : the same neurons as shown in $C$ but now the traces are aligned on the onset of the saccade. Each tick on the abscissa in each panel is separated $50 \mathrm{~ms}$. Arrows point to the visual transient occurring after the saccade. decrease in ROC area observed in SC neurons of the opposite hemisphere. Using the permutation test (see METHODS) we determined the time point when the separation between the stimulated and nonstimulated curves differed significantly for $\geq 5 \mathrm{~ms}$. For the crossed neurons we were able to determine a statistically significant separation time for 18 of $20(91 \%)$ neurons. The median time measured was $34.00 \mathrm{~ms}$ and the mean was $46.44 \mathrm{~ms}$ (Fig. 7B).

The red line in Fig. $7 A$ shows the ROC analysis result for the sample of uncrossed neurons. Initially there was a slight suppression in neuronal activity, as indicated in the ROC area that was slightly $<0.50$. Within about $100 \mathrm{~ms}$, the ROC area became slightly $>0.50$, indicating a slight increase in neuronal activity with $\mathrm{SNr}$ stimulation. Subsequently, there was a large reduction in ROC area. For the uncrossed neurons 20 of 22 $(91 \%)$ had statistically significant separation times. The median separation time measured was $73.50 \mathrm{~ms}$ and the mean was $77.55 \mathrm{~ms}$ (Fig. 7C). Comparing the separation times between the stimulated and unstimulated neuronal activity revealed a trend for the crossed SC neurons to have faster separation times compared with those of the uncrossed SC neurons (34.00 vs. $73.50 \mathrm{~ms}$ ), although this difference failed to reach statistical significance (Wilcoxon rank-sum test, $P=0.13$ ). The time course of the ROC area recapitulates the dynamics of the differences in response profiles of SC neurons that occurred with and without $\mathrm{SNr}$ stimulation (Figs. $7 A$ and 6, $A$ and $C$ ).

\section{SNr stimulation and saccade characteristics}

Our previous work using the stimulation parameters identical to those used here showed that $\mathrm{SNr}$ stimulation affected visually guided saccades only slightly, at least compared with memory-guided saccades (Basso and Liu 2007). Since we observed differences in SC neuronal activity around the time of the saccade-related burst, we explored whether saccades were altered in a predictable fashion. To assess changes in saccade parameters, we examined the saccades in the stimulation and no-stimulation conditions only for those data in which the SC neurons showed statistically significant ROC areas (18 crossed and 20 uncrossed).

We first explored alterations in the latency of saccades since our previous work revealed changes in saccade latency with stimulation of the SNr (Basso and Liu 2007). Since we observed changes in the tonic activity of $\mathrm{SC}$ neurons with $\mathrm{SNr}$ stimulation and this activity is associated with saccade latency (Basso and Wurtz 1998; Dorris et al. 1997), we reasoned that we should see predictable changes in saccade latency with $\mathrm{SNr}$ stimulation. Figure $8, A$ and $B$ shows the cumulative distributions of saccade latencies with and without $\mathrm{SNr}$ stimulation for uncrossed and crossed neurons. For the data obtained from uncrossed neurons (saccades contralateral to both the $\mathrm{SNr}$ and the SC), the mean latency of saccades without stimulation was $203.85 \mathrm{~ms}$, whereas the mean latency of saccades with stimulation was $184.67 \mathrm{~ms}$. The median latency without stimulation was $204 \mathrm{~ms}$, whereas the median latency with stimulation of the $\mathrm{SNr}$ was $185 \mathrm{~ms}$. These differences were statistically reliable (Fig. 8A, Wilcoxon rank-sum test, $P<0.001$ ).

For the data obtained from the crossed neurons (ipsilateral to the $\mathrm{SNr}$ but still contralateral to the SC), the mean saccade latency was $207.72 \mathrm{~ms}$, whereas the mean saccade latency with stimulation of the $\mathrm{SNr}$ was $211.11 \mathrm{~ms}$. The median latency without stimulation was $209 \mathrm{~ms}$, whereas the median latency with stimulation of the SNr was $207 \mathrm{~ms}$. These differences were statistically unreliable (Fig. $8 B$, Wilcoxon rank-sum test, $P=0.71)$. At the tails of the distribution there appeared to be 
TABLE 1. Distribution of SNr and SC neurons

\begin{tabular}{lll}
\hline \hline & \multicolumn{2}{c}{ Hemisphere } \\
\cline { 2 - 3 } & Crossed & Uncrossed \\
\hline
\end{tabular}

A. SNr neurons

Visual
Visual delay
Visual delay saccade
Visual saccade
Saccade
$\quad$ Total

$\begin{array}{rr}2 & 4 \\ 2 & 1 \\ 15 & 10 \\ 1 & 5 \\ 0 & 2 \\ 20 & 22\end{array}$

B. SC neurons

\begin{abstract}
Visuomotor (burst)
Buildup (prelude) Total
\end{abstract}

$\begin{array}{rr}2 & 2 \\ 18 & 20 \\ 20 & 22\end{array}$

C. Response field

\begin{tabular}{ccccc}
\multicolumn{2}{c}{ Overlapped } & & \multicolumn{2}{c}{ Nonoverlapped/Opposite } \\
\cline { 5 - 5 } Crossed & Uncrossed & & Crossed & Uncrossed \\
\hline $0 / 20$ & $18 / 22$ & $20 / 20$ & $4 / 22$ \\
\hline
\end{tabular}

The response characteristics of the $\mathrm{SC}$ and $\mathrm{SNr}$ neurons were determined as described in METHODS. SNr neuron types are listed and the numbers of each type indicated under the columns labeled "Crossed" or "Uncrossed." If the SNr neuron was located in the hemisphere opposite the recorded SC neuron, the $\mathrm{SNr}$ neuron type is indicated in the column labeled "Crossed." If the $\mathrm{SNr}$ neuron was located in the same hemisphere as the recorded SC neuron, the type is indicated in the column labeled "Uncrossed." A total of $20 \mathrm{SNr}$ stimulation sites and 20 SC neurons were from opposite sides of the brain (crossed). In all, $22 \mathrm{SNr}$ sites and $\mathrm{SC}$ neurons were stimulated and recorded from the same side of the brain (uncrossed). The SC neuron types are indicated and included in the columns labeled "Crossed" and "Uncrossed" according to the same criteria as for $\mathrm{SNr}$ neuron types. The same RFs indicate that the approximate centers of the $\mathrm{SNr}$ and SC neurons were located in the same hemifield. Nonoverlapping RFs indicate that the centers did not overlap. Most were located in the opposite hemifield.

differences in the saccade latency (Fig. 8B). If we divided the saccades into $>200$ - and $<200$-ms groups, the small differences between the nonstimulated and stimulated saccade latency distributions were statistically reliable (Fig. 8B, Kolmogorov-Smirnov, $P=0.04)$. Consistent with our previous observations (Basso and Liu 2007), stimulation of the $\mathrm{SNr}$ reduced the latency of saccades made contralateral to the stimulated $\mathrm{SNr}$ (uncrossed), whereas stimulation tended to increase the latency of saccades made ipsilateral to the stimulated $\mathrm{SNr}$ (crossed). Interestingly, this occurred despite the transient suppression of tonic activity of buildup neurons bilaterally.

Closer inspection of the saccades shown in Figs. 3, $A$ and $C$ and $4, A$ and $C$ suggested that the latency of the saccades not only became shorter or longer with stimulation of the $\mathrm{SNr}$, but also became more consistent on trials with $\mathrm{SNr}$ stimulation. To quantify a change in variability of saccade onset time and to determine whether this was a reliable observation across our sample of stimulation sites, we computed the SD of the saccade latency on trials with and without $\mathrm{SNr}$ stimulation. Across the sample of sites, the median of the SDs of saccade latencies on no-stimulation trials for uncrossed neurons was $31.75 \mathrm{~ms}$, whereas the median of the SDs on stimulated trials was 23.72 $\mathrm{ms}$. The difference in the medians of the SDs was statistically reliable (Wilcoxon rank-sum test, $P=0.03$ ). For the data collected during the crossed $\mathrm{SNr}$ stimulation-SC recordings (saccades ipsilateral to the $\mathrm{SNr}$ but still contralateral to the $\mathrm{SC}$ ), the median of the SDs of saccade latency was 28.33 without $\mathrm{SNr}$ stimulation, whereas the median of the SDs with $\mathrm{SNr}$ stimulation was 24.47. This smaller difference in saccade latency variability was not significant (Wilcoxon, $P=0.10$ ). Figure $8 C$ shows the means of the SDs of the saccade latencies for the 38 stimulation sites with significant ROC areas (see earlier text). In addition to decreases or increases in saccade latency with $\mathrm{SNr}$ stimulation, $\mathrm{SNr}$ stimulation in the same hemisphere as the SC responsible for generating the saccade reduced the variation in the onset time of saccades.
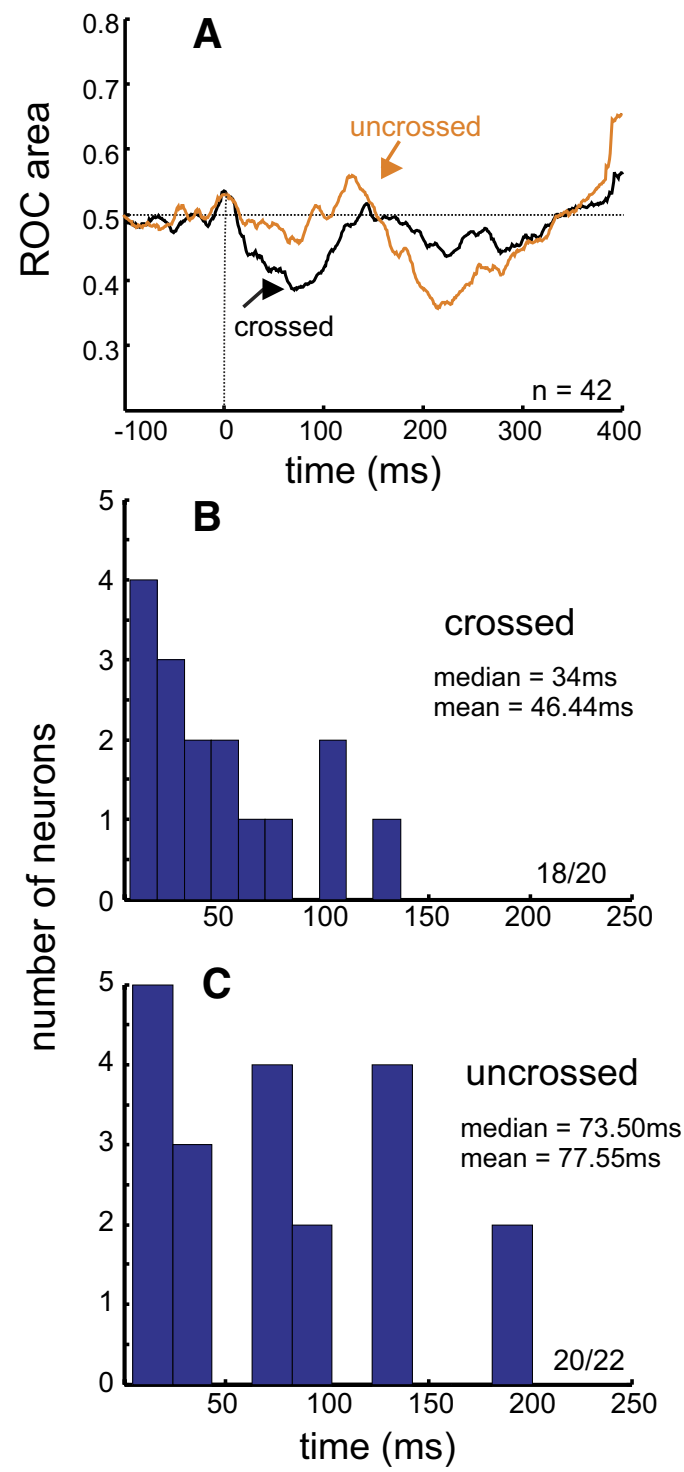

FIG. 7. Time course of $\mathrm{SNr}$ stimulation effects on $\mathrm{SC}$ neuronal activity. $A$ : receiver operating characteristic (ROC) area is plotted against time for all 42 SC neurons. The black line shows the result from the 20 crossed neurons; the red line shows the result from the 22 uncrossed neurons. The horizontal dashed line indicates a $0.50 \mathrm{ROC}$ area. (See text for description of how ROC area was calculated.) Values $<0.50$ indicate suppressed neuronal activity with $\mathrm{SNr}$ stimulation, whereas values $>0.50$ indicate enhanced neuronal activity with $\mathrm{SNr}$ stimulation. $B$ : frequency distribution of statistically significant separation times of neuronal activity recorded from crossed SC neurons; 18/20 crossed $\mathrm{SC}$ neurons had significant separation times. $C$ : the same as in $B$ except for SC neurons recorded on the same side as the $\mathrm{SNr}$ stimulation; 20/22 uncrossed SC neurons had significant separation times. 

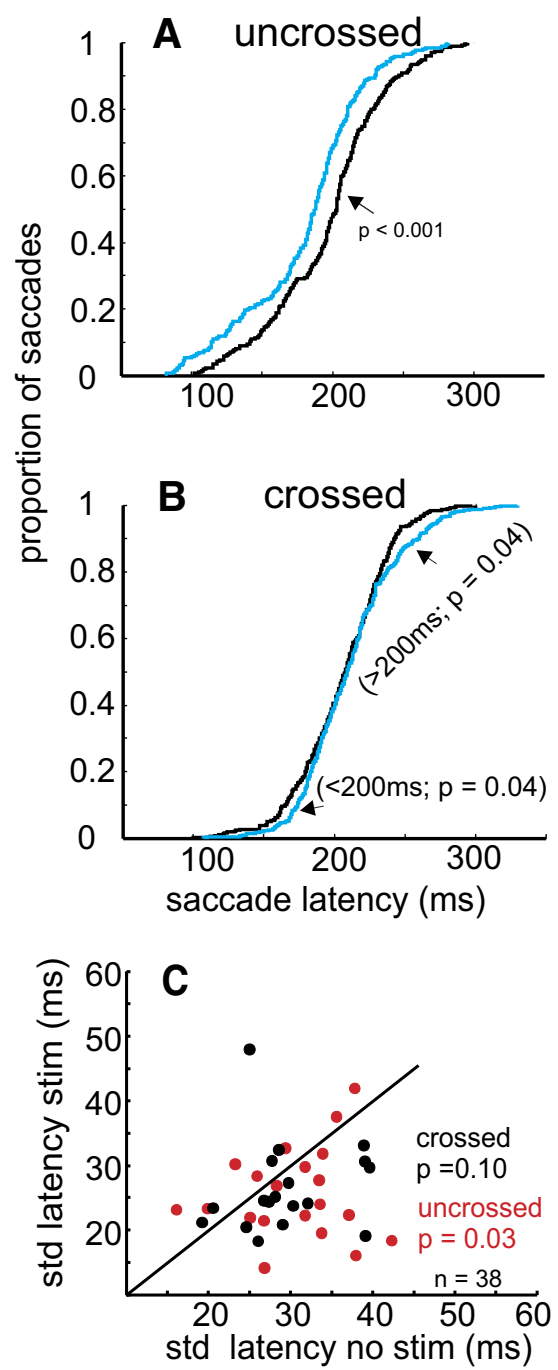

FIG. 8. SNr stimulation alters visually guided saccade latency. $A$ : the cumulative proportion of saccades across all stimulation sites is plotted against saccade latency (ms) for the data collected while recording from SC neurons located on the same side as the $\mathrm{SNr}$ stimulation (uncrossed). The black lines show the cumulative proportions for the no-stimulation trials. The blue lines show the cumulative proportions for the stimulation trials. $B$ : the same as in $A$ except for the trials in which the $\mathrm{SNr}$ stimulation occurred on the side contralateral to the recorded SC neuron. $C$ : the mean of the SDs of the saccade latencies measured across trials with stimulation of the SNr plotted against the mean of the SDs of the saccade latencies measured on trials without $\mathrm{SNr}$ stimulation. Each point is the mean computed from a single experiment and represents $\geq 10$ trials. The red circles show the data obtained from trials in which the SC neurons recorded were on the same side as the $\mathrm{SNr}$ stimulation (20 uncrossed neurons). The black circles show the data obtained from trials in which the SC neurons recorded were on the opposite side as the $\mathrm{SNr}$ stimulation (18 crossed neurons). The oblique dashed line is the line of unity. Points falling below the line indicate reduced SDs in the stimulated trials. Only the neurons with statistically significant changes in neuronal activity during $\mathrm{SNr}$ stimulation as determined by ROC and the permutation test (see text) are shown.

We also determined whether there were changes in the endpoints of saccades. We computed the radial amplitude and radial velocity of the eye movements made in the stimulation and no-stimulation trials, $r=\sqrt{ }\left(x^{2}+y^{2}\right)$, where $r$ is the radial amplitude or velocity, $x$ is the horizontal amplitude or velocity, and $y$ is the vertical amplitude or velocity. Figure 9 shows the distribution of saccade vectors in the nonstimulated (Fig. 9, $A$ and $B$, black arrows) and the stimulated trials (Fig. 9, $A$ and $B$, cyan arrows). The mean radial amplitude of the saccades made in no-stimulation trials was $10.88^{\circ}$ and in stimulation trials was $10.87^{\circ}$. These two distributions were statistically indistinguishable (Wilcoxon rank-sum test, $P=0.67$; data not shown). We obtained a similar result when comparing the radial velocity of the saccades made in stimulation trials and no-stimulation trials. The mean radial velocity of the saccades in no-stimulation trials was $523.80 \%$ and the mean radial velocity of the saccades made in stimulation trials was slightly increased to $528.00^{\circ} / \mathrm{s}$. These differences were statistically indistinguishable (Wilcoxon rank-sum test, $P=0.56$; data not shown). Thus despite statistically reliable changes in the neuronal discharge rate of SC neurons around the time of saccades, the endpoint amplitude and velocity of visually guided saccades remained unaltered with $\mathrm{SNr}$ stimulation.

Because we found changes in the variability of saccade latency with $\mathrm{SNr}$ stimulation we were also interested in whether there were more subtle changes in saccade characteristics such as in the variability of the amplitude or direction of the eye movement. We compared the SD of the saccade endpoint amplitudes across the sample of 38 sites. We found no significant effect of $\mathrm{SNr}$ stimulation on the amplitude variability of saccades (Wilcoxon rank-sum test; $P=0.15$ : crossed; $P=0.17$ : uncrossed). To determine whether there were changes in the variability of saccade direction, we computed the angle of the eye movement in degrees for the stimulated and nonstimulated trials and then determined the circular SD of the distribution of saccade directions (Fisher 1993). In contrast to the amplitude, the variability of the directions of the saccades differed with SNr stimulation. The median of the circular SDs measured on trials without stimulation was $2.32^{\circ}$, whereas the median of the circular SDs measured on trials with stimulation was $2.19^{\circ}$. This small decrease in variability was statistically significant (Wilcoxon rank-sum test, $P=0.01$ ). The change in variability was absent in saccades when the stimulation occurred in the crossed pathway (Wilcoxon rank-sum test, $P=0.21$ ). Thus as occurred with saccade latency, electrical stimulation of the $\mathrm{SNr}$ on the same side as the SC generating the saccade decreased the variability of saccade direction.

\section{I S C U S S I O N}

Our first goal was to test the hypothesis that electrical stimulation of the SNr influenced SC buildup neurons. Our second goal was to test the hypothesis that $\mathrm{SNr}$ influenced SC neurons bilaterally. Our combined stimulation and recording experiments provide direct evidence that the $\mathrm{SNr}$ influences buildup neurons bilaterally. The principal effect of electrical stimulation of the $\mathrm{SNr}$ was to suppress buildup neuronal activity within the SC of both hemispheres. We first discuss the relationship between our findings on SC buildup neurons and previous work on the uncrossed $\mathrm{SNr}-\mathrm{SC}$ pathway. We then discuss the relationship between our findings and previous work on the crossed $\mathrm{SNr}-\mathrm{SC}$ pathway. We then discuss the alterations in saccade characteristics and the implications for the role of the $\mathrm{SNr}-\mathrm{SC}$ pathway in saccade generation. 
Relationship to previous work on the uncrossed SNr-SC pathway

In the original studies of the ipsilateral $\mathrm{SNr}-\mathrm{SC}$ pathway in monkeys (Hikosaka and Wurtz 1983d), SNr neurons were driven antidromically with stimulation of the SC. SNr neurons were activated with short and fixed latencies, consistent with their known input to SC in multiple species (e.g., Chevalier et al. 1981; Graybiel 1978; Jayaraman et al. 1977). Furthermore, locations within the $\mathrm{SC}$ resulting in antidromic activation of $\mathrm{SNr}$ neurons occurred at multiple depths, suggesting that

\section{A uncrossed}

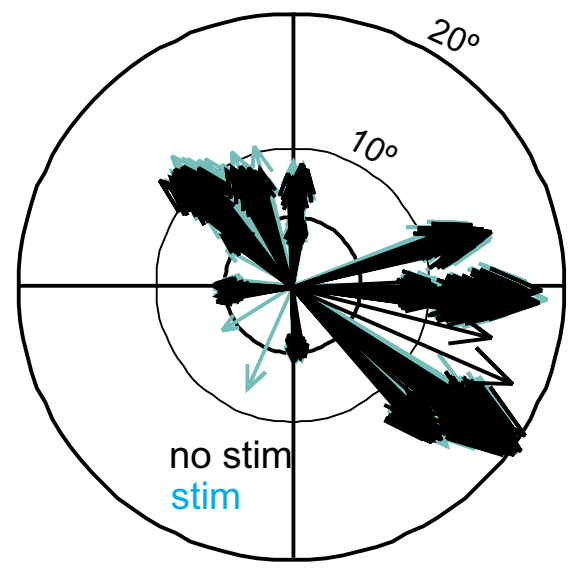

B crossed
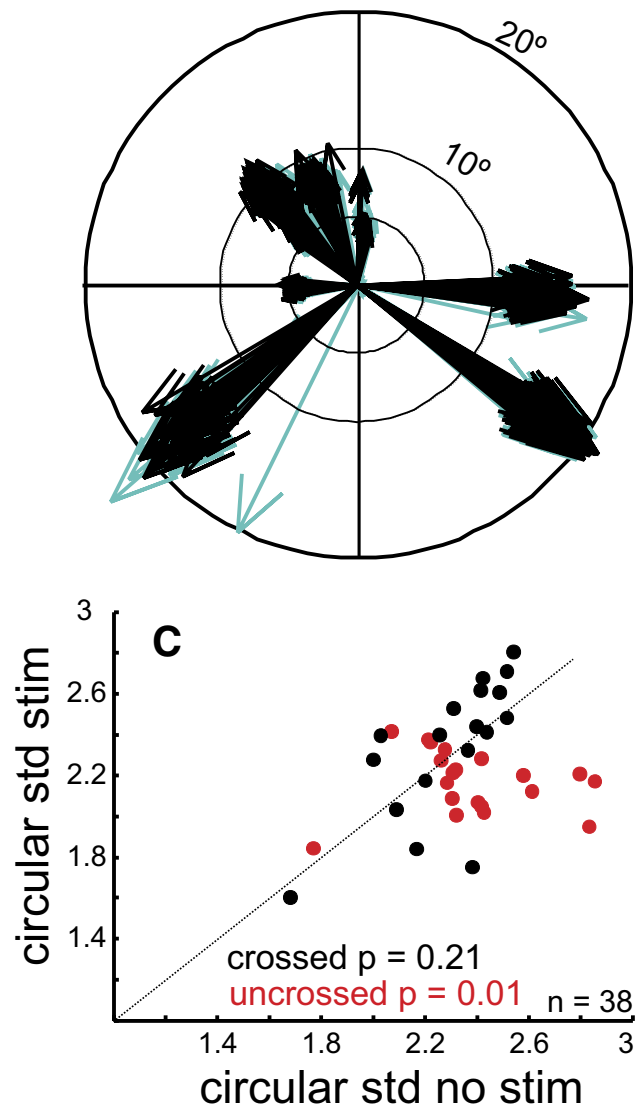

SNr axons branch extensively along the vertical dimension within the SC.

The work of Hikosaka and Wurtz (1983d) in the monkey emphasized the dorsolateral aspect of the SNr. This region provides input to the upper half of the intermediate layers of the SC, which is also presumably the location of the saccaderelated burst neurons (Harting et al. 1988; Huerta et al. 1991; May and Hall 1984; Munoz and Wurtz 1995). There is some suggestion that the buildup neurons are located below the burst neurons, in the lower part of the intermediate layers (Munoz and Wurtz 1995; Rodgers et al. 2006; Wurtz and Munoz 1994). We generally find burst and buildup neurons intermixed within the intermediate layers of the SC (Basso and Wurtz 1998; Krauzlis et al. 2000; Li and Basso 2005; Li et al. 2006). The present finding that $\mathrm{SNr}$ influences $\mathrm{SC}$ buildup neurons is consistent with the fact that we explored the $\mathrm{SNr}$ outside of the most dorsolateral region and with recent electrophysiological recordings in the monkey showing that neurons modulated during saccades and the occurrence of visual stimuli can be found throughout the extent of the SNr nucleus (Basso and Liu 2007; Handel and Glimcher 1999). Our result of SNr stimulation influencing buildup neurons also supports the hypothesis that the SNr influences many neuron types within the SC in addition to the saccade-related burst neurons (Karabelas and Moschovakis 1985). Future experiments will target other neurons within the SC such as the visual-tonic neurons, which generally are located more dorsally than saccade-related neurons in the SC (Li and Basso 2005; Li et al. 2006; McPeek and Keller 2002).

\section{Relationship to previous work on the crossed} SNr-SC pathway

A physiological demonstration of a contralateral SNr-SC pathway was recently identified in cats (Jiang et al. 2003), but as far as we are aware, such a pathway has not been explored systematically in the monkey (Karabelas and Moschovakis 1985). The current conceptual model of the role of the SNr-SC in saccade generation in the monkey emphasizes the uncrossed $\mathrm{SNr}-\mathrm{SC}$ projection, yet there is anatomical evidence in rodents, cats, and monkeys that the SNr targets the SC bilaterally (Deniau and Chevalier 1992; Gerfen et al. 1982; Jayaraman et al. 1977; Jiang et al. 2003; Redgrave et al. 1992). Our results showing that stimulation of the SNr influences buildup neurons in the SC on the opposite side of the brain provide further, direct evidence for a crossed $\mathrm{SNr}-\mathrm{SC}$ pathway in monkeys.

FIG. 9. SNr stimulation alters visually guided saccade direction. $A$ : saccade vectors are indicated by the arrows. Black arrows are no-stimulation trials and blue arrows are stimulation trials. Each saccade recorded from each trial and each experiment is plotted in polar coordinates for the uncrossed data. $B$ : the same as in $A$ for the crossed data. The direction of each arrow indicates the saccade direction and thus the preferred direction of the SC neuron for that recording day. Note that multiple experiments may have had multiple similar directions. $C$ : the circular SD of saccade directions measured across trials with $\mathrm{SNr}$ stimulation is plotted against the circular SD of saccade directions measured across trials without $\mathrm{SNr}$ stimulation. The red circles show the data obtained from trials in which the SC neurons recorded were on the same side as the $\mathrm{SNr}$ stimulation (20 uncrossed neurons). The black circles show the data obtained from trials in which the SC neurons recorded were on the opposite side as the SNr stimulation ( 18 crossed neurons). The oblique dashed line is the line of unity. Points falling below the line indicate a reduced SD in the stimulated trials. Only the neurons with statistically significant suppression of neuronal activity during $\mathrm{SNr}$ stimulation as determined by $\mathrm{ROC}$ and the permutation test (see text) are shown. 
Also, the current model of the $\mathrm{SNr}-\mathrm{SC}$ involvement in saccades focuses on the transient pause around the time of a contralateral saccade (with respect to the $\mathrm{SNr}$ ) and the temporally correlated increase in discharge of burst neurons in the SC (Hikosaka et al. 2006; Lo and Wang 2006). However, neurons in the cat and monkey $\mathrm{SNr}$ display a variety of response profiles around the time of head or eye movements and the onset of visual stimuli both ipsilaterally and contralaterally (Basso and Wurtz 2002; Basso et al. 2005; Bayer et al. 2002; Boussaoud and Joseph 1985; Handel and Glimcher 1999, 2000; Hikosaka and Wurtz 1983a,b,c). Taken together, the model of the role of the $\mathrm{SNr}$ in saccades should be revisited.

In the cat, two distinct neuronal populations in the $\mathrm{SNr}$ contribute to the crossed and uncrossed pathways to the SC (Jiang et al. 2003). Characteristics of uncrossed SNr neurons include a broad distribution throughout the extent of the $\mathrm{SNr}$ nucleus, lower conduction velocities compared with crossed $\mathrm{SNr}$ neurons, discharge rates ranging between 11 and 86 spikes/s, RF locations centered on the contralateral hemifield, RF sizes smaller than those of crossed pathway neurons, and transient pauses in tonic activity associated with the onset of visual stimuli. Crossed neurons, in contrast, are located within the anterolateral portion of the $\mathrm{SNr}$, have higher conduction velocities compared with those of uncrossed neurons, discharge rates ranging between 0.12 and 40 spikes/s, have large RFs with locations centered on the ipsilateral hemifield, and transient increases in neuronal discharge associated with a visual stimulus moving through the RF. Based on these differences, and the finding that both uncrossed and crossed $\mathrm{SNr}$ neurons receive input from prestriate visual cortex, the authors suggested that the crossed and uncrossed pathways work in concert to produce a wanted movement through disinhibition of the SC on the same side, while simultaneously preventing an unwanted movement by inhibition of the SC on the opposite side.

Our results in the monkey are consistent with the cat results. For example, in the cat, using orthodromic stimulation, a $100-\mu \mathrm{s}, 100-$ to $300-\mu \mathrm{A}$ stimulation pulse to the $\mathrm{SNr}$ suppressed the visual response of SC neurons recorded on the opposite side of the brain $(n=4)$ (Jiang et al. 2003). Although we used trains of electrical stimulation $(60 \mu \mathrm{A}, 150 \mu \mathrm{s}, 300$ $\mathrm{Hz}, 400-\mathrm{ms}$ duration), we found a similar suppression of SC activity, indicating that the crossed $\mathrm{SNr}-\mathrm{SC}$ pathway exerts inhibition on SC neurons like its uncrossed counterpart. However, a number of differences are evident between the cat results and the monkey results reported here.

First, in general, $\mathrm{SNr}$ sites of stimulation influencing the same or opposite SC were intermingled within the $\mathrm{SNr}$ nucleus. We did not find an obvious segregation between crossed and uncrossed $\mathrm{SNr}-\mathrm{SC}$ neurons. Indeed, an inability to drive $\mathrm{SNr}$ neurons antidromically does not indicate conclusively that the input does not exist. The stimulating electrodes in the cat experiments were located along a rostrocaudal axis. Perhaps antidromic activation would have been obtained had the stimulating electrodes been oriented along the mediolateral axis. Anecdotal reports in the cat suggest that single SC neurons are inhibited equally well from either $\mathrm{SNr}$ (Karabelas and Moschovakis 1985). We report here 13 SC neurons that were inhibited by stimulation from either SNr. If the monkey crossed and uncrossed pathways arise from segregated neuronal pools within the $\mathrm{SNr}$ as antidromic experiments in the cat suggest, then our ability to influence the same SC from either $\mathrm{SNr}$ must have resulted from systematically stimulating two different regions of the SNr. Although possible, we think this is unlikely. Second, the great majority of our $\mathrm{SNr}$ neurons had tonic discharge rates in excess of 40 spikes/s. We did not find a systematic difference in discharge rate of neurons recorded at sites in the $\mathrm{SNr}$ where stimulation influenced the opposite SC. Third, despite the profound suppression of SC activity, the saccade-related burst and the resulting saccade still occurred in our experiments with $\mathrm{SNr}$ stimulation. This result is not predicted from the cat results.

There are at least two possible explanations for the finding that the SC burst was only scarcely affected by the $\mathrm{SNr}$ stimulation. The first possibility is that the stimulation train used in our experiments depleted $\gamma$-aminobutyric acid or saturated postsynaptic receptors and thus was no longer effective at suppressing SC activity. We think this is unlikely because when we realigned the traces to the onset of the saccade, we found that suppression of SC activity, although weak, extended for most of the train duration (Fig. 6). A second possibility is that the combination of the persistent visual drive to the SC, likely arising from extrastriate cortex (eg.,Paré and Wurtz 2001), and the recurrent excitation among pools of SC neurons (Moschovakis et al. 1996; Özen et al. 2004) was able to override the inhibition arising from SNr. If this is true, stimulation of the $\mathrm{SNr}$ in the absence of a visual drive should be maximally effective on SC neurons. Consistent with this hypothesis, we previously found that stimulation of the $\mathrm{SNr}$ affected memory-guided saccades more profoundly than visually guided saccades (Basso and Liu 2007). Because of the known "upward shift" of memory-guided saccades (Gnadt and Andersen 1988; White et al. 1994) and the differences in discharge among SC neurons for memory-guided saccades (Stanford and Sparks 1994), we did not test memory saccades in the present experiments. Therefore future experiments will have to be performed to determine whether a greater effect of $\mathrm{SNr}$ stimulation exists on SC neurons for saccades made to locations without visual stimuli present.

A final difference between our results and those reported in the cat is that in our sample of $\mathrm{SNr}$ neurons the most common response profile was a decrease in activity (data not shown). In the cat, $\mathrm{SNr}$ neurons projecting to the same side SC showed pauses in neuronal activity and the $\mathrm{SNr}$ neurons projecting to the opposite SC showed increases in neuronal activity for the appearance of visual stimuli. In the sample of $\mathrm{SNr}$ neurons reported here, 5 of $42 \mathrm{SNr}$ neurons might fall within the category described by Handel and Glimcher (1999) as pause bursters. At the sites where three of these were recorded, stimulation of the $\mathrm{SNr}$ influenced the $\mathrm{SC}$ in the opposite hemisphere. Thus based on this limited sample, we find $\mathrm{SNr}$ neuronal response properties intermingled within the nucleus and we find no clear difference in the neuronal response types located at sites of stimulation within the $\mathrm{SNr}$ capable of influencing SC activity.

\section{Relationship of stimulation effects to saccade behavior}

A surprising finding reported here was the overall small effect of stimulation on the amplitude and velocity of the saccades. Also surprising was the large decrease observed for contralateral saccade latency, in spite of the decreases in SC 
buildup neuronal activity. Surprising and in our view, most interesting, was the observation that the variability in saccade latency and direction was reduced with stimulation of the SNr. Combining these results suggests that the influence of the $\mathrm{SNr}$ on the SC and visually guided saccade behavior is more subtle than previously thought. We first discuss the changes in saccade latency and then discuss the changes in latency and direction variability.

Across the sample of neurons reported here, we found reliable decreases in discharge rate of $\mathrm{SC}$ neurons with $\mathrm{SNr}$ stimulation. We also found very reliable decreases in contralateral saccade latency with $\mathrm{SNr}$ stimulation. There are at least two explanations for this. First, electrical stimulation of the SNr may have activated descending corticofugal fibers exciting the SC (Chevalier et al. 1984; Karabelas and Moschovakis 1985). Second, stimulation of the SNr could antidromically activate cholinergic peduncolopontine neurons (Scarnati et al. 1984). Activation of pedunculopontine neurons would excite SC saccade-related neurons (Watanabe et al. 2005). Consistent with this, we initially found in the uncrossed SC neurons a lower level of suppression of neuronal activity, compared with that seen in the crossed SC neurons. This may indicate that both excitatory and inhibitory drives were activated in the uncrossed pathway. Certainly, local injections of glutamate into the $\mathrm{SNr}$ combined with recording of SC neurons will be required to determine conclusively whether the decreases in latency depend on activation of fibers of passage.

Another possibility that could explain the decrease in contralateral saccade latency is that $\mathrm{SNr}$ neurons that project to the buildup neurons also have a collateral axon that targets an inhibitory interneuron within the SC. In this scheme, stimulation of the $\mathrm{SNr}$ would produce an initial inhibition of the buildup neuron through its direct input and then a subsequent disinhibition arising from the collateral input to the inhibitory interneuron. The initial suppression followed by the apparent faster rise of the saccade-related burst are consistent with this idea (Fig. 6A). We favor this hypothesis over the hypothesis that the stimulation activated excitatory inputs for three reasons. One, if the stimulation activated the excitatory input we should have observed increases in SC neuronal activity more often than we did. Only three neurons showed only modest increases in activity with $\mathrm{SNr}$ stimulation in our sample. Second, recent in vitro work in the rodent has identified a projection from the $\mathrm{SNr}$ to GAD+ inhibitory interneurons within the SC (Yanagawa et al. 2007). Third, based on our previous results, $\mathrm{SNr}$ stimulation affects memory saccades more than visually guided saccades (Basso and Liu 2007). If the effects of stimulation resulted primarily from activation of excitatory inputs to the SC, the effects on saccades should be independent of context.

It is interesting that the decreases in saccade latency as well as the decreases in variability of saccade latency and saccade direction all occurred with stimulation of the $\mathrm{SNr}$ on the same side as the SC generating the saccade. It is not immediately obvious how these changes would result from activation of an excitatory drive. Rather, we think these kinds of changes in behavior are more easily understood with disinhibition, as would occur if the SNr targets an inhibitory interneuron that also influences buildup neurons. Regardless of the mechanism, the result is consistent with the hypothesis that the influence of the $\mathrm{SNr}$ on $\mathrm{SC}$ is not to gate the occurrence of saccades-at least visually guided saccades-in an all-or-none fashion. Rather, the results suggest that the input from the SNr to the SC plays a more subtle role (Hikosaka et al. 2000), perhaps by altering the balance of excitatory drives arising from cortex and the inhibitory influences arising from intrinsic circuits.

\section{A C K N OW LED G M EN T S}

We thank Dr. Xiaobing Li, B. Kim, and Dr. Xu Yang for assistance with data collection and B. Kim for Matlab code. We also thank the Parkinson Disease Foundation for funding the summer student fellowship of T.J.G. Day, who participated in preliminary data collection and analysis. We also thank the anonymous reviewers for helpful comments on the manuscript.

\section{G R A N T S}

This work was supported by National Eye Institute Grant EY-13692 and the Esther A. and Joseph Klingenstein Foundation to M. A. Basso, and National Center for Research Resources Grant P51 RR-000167 to the Wisconsin National Primate Research Center.

\section{REFERENCES}

Anderson ME, Postupna N, Ruffo M. Effects of high-frequency stimulation in the internal globus pallidus on the activity of thalamic neurons in the awake monkey. J Neurophysiol 89: 1150-1160, 2003.

Asanuma H, Arnold AP. Noxious effects of excessive currents used for intracortical microstimulation. Brain Res 96: 103-107, 1975.

Basso MA, Liu P. Context-dependent effects of substantia nigra stimulation on eye movements. J Neurophysiol 97: 4129-4142, 2007.

Basso MA, Pokorny JJ, Liu P. Activity of monkey substantia nigra pars reticulata neurons during smooth pursuit eye movements. Eur J Neurosci 22: 448-464, 2005.

Basso MA, Wurtz RH. Modulation of neuronal activity by target uncertainty. Nature 389: 66-69, 1997.

Basso MA, Wurtz RH. Modulation of neuronal activity in superior colliculus by changes in target probability. J Neurosci 18: 7519-7534, 1998.

Basso MA, Wurtz RH. Neuronal activity in substantia nigra pars reticulata during target selection. J Neurosci 22: 1883-1894, 2002.

Bayer HM, Handel A, Glimcher PW. Eye position and memory saccade related responses in substantia nigra pars reticulata. Exp Brain Res 154: 428-441, 2002.

Beckstead RM. Long collateral branches of substantia nigra pars reticulata axons to thalamus, superior colliculus and reticular formation in monkey and cat. Multiple retrograde neuronal labeling with fluorescent dyes. Neuroscience 10: 767-779, 1983.

Bishop PO, Burke W, Davis R. Single-unit recording from antidromically activated optic radiation neurones. J Physiol 162: 432-450, 1962.

Boussaoud D, Joseph JP. Role of the cat substantia nigra pars reticulata in eye and head movements. II. Effects of local pharmacological injections. Exp Brain Res 57: 297-304, 1985.

Bradley A, Skottun BC, Ohzawa I, Sclar G, Freeman RD. Visual orientation and spatial frequency discrimination: a comparison of single neurons and behavior. J Neurophysiol 57: 755-772, 1987.

Britten KH, Shadlen MN, Newsome WT, Movshon JA. The analysis of visual motion: a comparison of neuronal and psychophysical performance. J Neurosci 12: 4745-4565, 1992.

Chevalier G, Deniau JM. Disinhibition as a basic process in the expression of striatal functions. Trends Neurosci 13: 277-280, 1990.

Chevalier G, Thierry AM, Shibazaki T, Feger J. Evidence for a GABAergic inhibitory nigrotectal pathway in the rat. Neurosci Lett 21: 67-70, 1981.

Chevalier G, Vacher S, Deniau JM. Inhibitory nigral influence on tectospinal neurons, a possible implication of basal ganglia in orienting behavior. Exp Brain Res 53: 320-326, 1984.

Chevalier G, Vacher S, Deniau JM, Desban M. Disinhibition as a basic process in the expression of striatal functions. I. The striato-nigral influence on tecto-spino/tecto-diencephalic neurons. Brain Res 334: 215-226, 1985.

Cohn TE, Green DG, Tanner WJ. Receiver operating characteristic analysis: application to the study of quantum fluctuation effects in optic nerve of Rana pipiens. J Gen Physiol 66: 583-616, 1975.

Comoli E, Coizet V, Boyes J, Bolam PJ, Canteras NS, Quirk RH, Overton PG, Redgrave P. A direct projection from the superior colliculus to substantia nigra for detecting salient visual events. Nat Neurosci 6: $974-$ 980, 2003. 
Crist CF, Yamasaki DSG, Komatsu H, Wurtz RH. A grid system and a microsyringe for single cell recording. J Neurosci Methods 26: 117-122, 1988.

Deniau JM, Chevalier G. The lamellar organization of the rat substantia nigra pars reticulata: distribution of projection neurons. Neuroscience 46: 361377, 1992.

Dorris MC, Munoz DP. Saccadic probability influences motor preparation signals and time to saccadic initiation. J Neurosci 18: 7015-7026, 1998.

Dorris MC, Paré M, Munoz DP. Neuronal activity in monkey superior colliculus related to the initiation of saccadic eye movements. J Neurosci 17 : 8566-8579, 1997.

Efron B, Tibshirani RJ. An Introduction to the Bootstrap. Washington, DC: Chapman \& Hall/CRC Press, 1998.

Fisher NI. Statistical Analysis of Circular Data. Cambridge, UK: Cambridge Univ. Press, 1993.

François C, Percheron G, Yelnik J. Localization of nigrostriatal, nigrothalamic and nigrotectal neurons in ventricular coordinates in macaques. Neuroscience 13: 61-76, 1984.

Fries W. Cortical projections to the superior colliculus in the macaque monkey: a retrograde study using horseradish peroxidase. J Comp Neurol 230: 55-76, 1984.

Fuchs AF, Robinson DA. A method for measuring horizontal and vertical eye movement chronically in the monkey. J Appl Physiol 21: 1068-1070, 1966.

Fuller JH, Schlag JD. Determination of antidromic excitation by the collision test: problems of interpretation. Brain Res 112: 283-298, 1976.

Gerfen CR, Staines WA, Arbuthnott GW, Fibiger HC. Crossed connections of the substantia nigra in the rat. J Comp Neurol 207: 283-303, 1982.

Glimcher PW, Sparks DL. Movement selection in advance of action in the superior colliculus. Nature 355: 542-545, 1992.

Gnadt JW, Andersen RA. Memory related motor planning activity in posterior parietal cortex of macaque. Exp Brain Res 70: 216-220, 1988.

Gnadt JW, Echols SD, Yildirim A, Zhang H, Paul K. Spectral cancellation of microstimulation artifact for simultaneous neural recording in situ. IEEE Trans Biomed Eng 50: 1129-1135, 2003

Graybiel AM. Organization of the nigrotectal connection: an experimental tracer study in the cat. Brain Res 143: 339-348, 1978.

Green DM, Swets JA. Signal Detection Theory and Psychophysics. New York: Wiley, 1966.

Handel A, Glimcher PW. Quantitative analysis of substantia nigra pars reticulata activity during a visually guided saccade task. J Neurophysiol 82: 3458-3475, 1999

Handel A, Glimcher PW. Contextual modulation of substantia nigra pars reticulata neurons. J Neurophysiol 83: 3042-3048, 2000

Harting JK, Huerta MF, Hashikawa T, Weber JT, Van Lieshout DP. Neuroanatomical studies of the nigrotectal projections in the cat. J Comp Neurol 278: 615-631, 1988.

Harting JK, Updyke BV, Van Lieshout DP. Corticotectal projections in the cat: anterograde transport studies of twenty-five cortical areas. J Comp Neurol 324: 379-414, 1992.

Hikosaka O, Nakamura K, Nakahara H. Basal ganglia orient eyes to reward. J Neurophysiol 95: 567-584, 2006

Hikosaka O, Sakamoto M, Miyashita N. Effects of caudate nucleus stimulation on substantia nigra cell activity in monkey. Exp Brain Res 95: 457-472, 1993.

Hikosaka O, Takikawa Y, Kawagoe R. Role of the basal ganglia in the control of purposive saccadic eye movements. Physiol Rev 80: 953-978, 2000 .

Hikosaka O, Wurtz RH. Visual and oculomotor functions of monkey substantia nigra pars reticulata. I. Relation of visual and auditory responses to saccades. J Neurophysiol 49: 1230-1253, 1983 a.

Hikosaka O, Wurtz RH. Visual and oculomotor functions of monkey substantia nigra pars reticulata. II. Visual responses related to fixation of gaze. J Neurophysiol 49: 1254-1267, 1983b.

Hikosaka O, Wurtz RH. Visual and oculomotor functions of monkey substantia nigra pars reticulata. III. Memory-contingent visual and saccade responses. J Neurophysiol 49: 1268-1284, 1983c.

Hikosaka O, Wurtz RH. Visual and oculomotor functions of monkey substantia nigra pars reticulata. IV. Relation of substantia nigra to superior colliculus. J Neurophysiol 49: 1285-1301, 1983d.

Hikosaka O, Wurtz RH. Modification of saccadic eye movements by GABArelated substances. I. Effect of muscimol and bicuculline in monkey superior colliculus. J Neurophysiol 53: 266-291, 1985a
Hikosaka O, Wurtz RH. Modification of saccadic eye movements by GABArelated substances. II. Effects of muscimol in monkey substantia nigra pars reticulata. J Neurophysiol 53: 292-308, 1985 b.

Horwitz GD, Newsome WT. Separate signals for target selection and movement specification in the superior colliculus. Science 284: 1158-1161, 1999.

Horwitz GD, Newsome WT. Target selection for saccadic eye movements: prelude activity in the superior colliculus during a direction-discrimination task. J Neurophysiol 86: 2543-2558, 2001.

Huerta MF, Van Lieshout DP, Harting JK. Nigrotectal projections in the primate Galago crassicaudatus. Exp Brain Res 87: 389-401, 1991.

Ignashchenkova A, Dicke PW, Haarmeier T, Thier P. Neuron-specific contribution of the superior colliculus to overt and covert shifts of attention. Nat Neurosci 7: 56-64, 2004.

Jayaraman A, Batton RR 3rd, Carpenter MB. Nigrotectal projections in the monkey: an autoradiographic study. Brain Res 135: 147-152, 1977.

Jiang H, Stein BE, McHaffie JG. Opposing basal ganglia processes shape midbrain visuomotor activity bilaterally. Nature 423: 982-986, 2003.

Joseph JP, Boussaoud D. Role of the cat substantia nigra pars reticulata in eye and head movements. I. Neural activity. Exp Brain Res 57: 286-296, 1985.

Judge SJ, Richmond BJ, Chu FC. Implantation of magnetic search coils for measurement of eye position: an improved method. Vision Res 20: 535-538, 1980.

Karabelas AB, Moschovakis AK. Nigral inhibitory termination on efferent neurons of the superior colliculus: an intracellular horseradish peroxidase study in the cat. J Comp Neurol 239: 309-329, 1985.

Keppel G. Design and Analysis: A Researcher's Handbook. Upper Saddle River, NJ: Prentice Hall, 1991.

Kim B, Basso MA. Saccade target selection: a signal detection theory approach. J Neurosci 28: 2991-3007, 2008.

Krauzlis RJ, Basso MA, Wurtz RH. Discharge properties of neurons in the rostral superior colliculus of the monkey during smooth-pursuit eye movements. J Neurophysiol 84: 876-891, 2000.

Krauzlis RJ, Dill N. Neural correlates of target choice for pursuit and saccades in the primate superior colliculus. Neuron 35: 355-363, 2002.

Kustov AA, Robinson DL. Shared neural control of attentional shifts and eye movements. Nature 384: 74-77, 1996.

Li X, Basso MA. Competitive stimulus interactions within single response fields of superior colliculus neurons. J Neurosci 25: 11357-11373, 2005.

Li X, Kim B, Basso MA. Transient pauses in delay-period activity of superior colliculus neurons. J Neurophysiol 95: 2252-2264, 2006.

Lo C-C, Wang X-J. Cortico-basal ganglia circuit mechanism for a decision threshold in reaction time tasks. Nat Neurosci 9: 956-963, 2006.

Ma TP. Identification of the substantia nigra pars lateralis in the macaque using cytochrome oxidase and fiber stains. Brain Res 480: 305-311, 1989.

May PJ, Hall WC. Relationships between the nigrotectal pathway and the cells of origin of the predorsal bundle. J Comp Neurol 226: 357-376, 1984.

McPeek RM, Keller EL. Saccade target selection in the superior colliculus during a visual search task. J Neurophysiol 88: 2019-2034, 2002.

Moschovakis AK, Scudder CA, Highstein SM. The microscopic anatomy and physiology of the mammalian saccadic system. Prog Neurobiol 50: 133-254, 1996.

Munoz DP, Wurtz RH. Saccade-related activity in monkey superior colliculus. I. Characteristics of burst and buildup cells. J Neurophysiol 73: 2313 2333, 1995

Özen G, Helms MC, Hall WC. The intracollicular neuronal network. In: The Superior Colliculus: New Approaches for Studying Sensorimotor Integration, edited by Hall WC, Moschovakis AK. Boca Raton, FL: CRC Press, 2004, p. 147-158.

Paré M, Wurtz RH. Progression in neuronal processing for saccadic eye movements from parietal cortex area LIP to superior colliculus. J Neurophysiol 85: 2545-2562, 2001.

Parthasarathy HB, Schall JD, Graybiel AM. Distributed but convergent ordering of corticostriatal projections: analysis of frontal eye field and supplementary eye field in the macaque monkey. J Neurosci 12: $4468-$ 4488, 1992.

Paul K, Gnadt JW. Reliable real-time spike discrimination during microstimulation. J Neurosci Methods 128: 191-195, 2003.

Ratcliff R, Cherian A, Segraves M. A comparison of macaque behavior and superior colliculus neuronal activity to predictions from models of two choice decisions. J Neurophysiol 90: 1392-1407, 2003.

Redgrave P, Marrow L, Dean P. Topographical organization of the nigrotectal projection in rat: evidence for segregated channels. Neuroscience 50: 571-595, 1992 
Rodgers CK, Munoz DP, Scott SH, Paré M. Discharge properties of monkey tectoreticular neurons. J Neurophysiol 95: 3502-3511, 2006.

Sato M, Hikosaka O. Role of primate substantia nigra pars reticulata in reward-oriented saccadic eye movement. J Neurosci 22: 2363-2373, 2002.

Scarnati E, Campana E, Pacitti C. Pedunculopontine-evoked excitation of substantia nigra neurons in the rat. Brain Res 304: 351-361, 1984.

Segraves MA, Goldberg ME. Functional properties of corticotectal neurons in the monkey's frontal eye field. J Neurophysiol 58: 1387-1419, 1987.

Selemon LD, Goldman-Rakic PS. Longitudinal topography and interdigitation of corticostriatal projections in the rhesus monkey. J Neurosci 5: 776-794, 1985.

Selemon LD, Goldman-Rakic PS. Common cortical and subcortical targets of the dorsolateral prefrontal and posterior parietal cortices in the rhesus monkey: evidence for a distributed neural network subserving spatially guided behavior. J Neurosci 8: 4049-4068, 1988.

Sommer MA, Wurtz RH. Composition and topographic organization of signals sent from the frontal eye field to the superior colliculus. J Neurophysiol 83: 1979-2001, 2000.

Sparks DL. Response properties of eye movement-related neurons in the monkey superior colliculus. Brain Res 90: 147-152, 1975.

Stanford TR, Sparks DL. Systematic errors for saccades to remembered targets: evidence for a dissociation between saccade metrics and activity in the superior colliculus. Vision Res 34: 93-106, 1994.

Stanton GB, Bruce CJ, Goldberg ME. Frontal eye field efferents in the macaque monkey. I. Subcortical pathways and topography of striatal and thalamic terminal fields. J Comp Neurol 271: 473-492, 1988a.
Stanton GB, Bruce CJ, Goldberg ME. Frontal eye field efferents in the macaque monkey. II. Topography of terminal fields in midbrain and pons. J Comp Neurol 271: 493-506, 1988b.

Thompson KG, Hanes DP, Bichot NP, Schall JD. Perceptual and motor processing stages identified in the activity of macaque frontal eye field neurons during visual search. J Neurophysiol 76: 4040-4054, 1996.

Watanabe M, Kobayashi Y, Inoue Y, Isa T. Effects of local nicotinic activation of the superior colliculus on saccades in monkeys. J Neurophysiol 93: 519-534, 2005.

Weyand TG, Gafka AC. Corticostriatal and corticotectal neurons in area 6 of the cat during fixation and eye movements. Vis Neurosci 15: 141-151, 1998.

White JM, Sparks DL, Stanford TR. Saccades to remembered target locations: an analysis of systematic and variable errors. Vision Res 34: 79-92, 1994.

Wurtz RH, Munoz DP. Organization of saccade related neurons in monkey superior colliculus. In: Contemporary Ocular Motor and Vestibular Research: A Tribute to David A. Robinson, edited by Büttner U, Brandt T, Fuchs A, Zee D. Stuttgart, Germany: Thieme, 1994, p. 520-527.

Yanagawa Y, K K, Isa K, Obata K, Isa T. Direct nigral inhibitory inputs to GABAergic neurons in mouse superior colliculus. Program 718.1. 2007 Abstract Viewer/Itinerary Planner. San Diego, CA: Society for Neuroscience, 2007. CD-ROM.

York DH, Faber JE. An electrophysiological study of nigro-tectal relationships: a possible role in turning behavior. Brain Res 130: 383-386, 1977. 